Original paper

\title{
Simulation of proton range monitoring in an anthropomorphic phantom using multi-slat collimators and time-of-flight detection of prompt-gamma quanta
}

\author{
Patricia Cambraia Lopes ${ }^{\mathrm{a}, \mathrm{b}, \mathrm{c}}$, Paulo Crespo ${ }^{\mathrm{b}, \mathrm{d}, *}$, Hugo Simões ${ }^{\mathrm{b}, \mathrm{d}}$, Rui Ferreira Marques ${ }^{\mathrm{b}, \mathrm{d}}$, \\ Katia Parodi ${ }^{\mathrm{c}, \mathrm{e}}$, Dennis R Schaart ${ }^{\mathrm{a}, \mathrm{f}}$

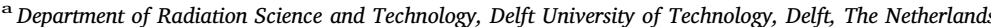 \\ b Laboratório de Instrumentação e Física Experimental de Partículas, Coimbra, Portugal \\ ${ }^{\mathrm{c}}$ Heidelberg Ion-Beam Therapy Center, Heidelberg University Clinic, Heidelberg, Germany \\ d Physics Department, Universidade de Coimbra, Coimbra, Portugal \\ e Department of Medical Physics, Ludwig-Maximilians-Universität München, Munich, Germany \\ ${ }^{\mathrm{f}}$ Holland Proton Therapy Centre (Holland PTC), Delft, The Netherlands
}

\section{A R T I C L E I N F O}

\section{Keywords:}

Prompt gamma imaging

Multi-slat collimator

Shifting time-of-flight

Anthropomorphic phantom

\begin{abstract}
A B S T R A C T
Prompt-gamma (PG) imaging has the potential for monitoring proton therapy in real time. Different approaches are investigated. We focus on developing multi-slat collimators to image PG quanta, aiming at optimizing collimator performance to detect deviations in treatment delivery. We investigated six different multi-slat configurations, which have either optimal (analytical) intrinsic spatial resolution at fixed efficiency, or otherwise; at different distances from the proton pencil-beam axis $(15 \mathrm{~cm}-35 \mathrm{~cm})$. We used Geant 4 to simulate irradiations of the head (energy: $130 \mathrm{MeV}$ ) and pelvis (200 MeV) of an anthropomorphic phantom, with and without physiologic/morphologic or setup changes of clinical dosimetric relevance. The particles escaping the phantom were transported through each of these multi-slat configurations and the gamma counts profiles were recorded at the collimator exit. Median filtering was applied to the registered PG-profiles to mitigate the effects of septa shadowing and statistical fluctuations. Time-of-flight discrimination was used to enhance the signal-to-background ratio, which appeared crucial for $200 \mathrm{MeV}$ irradiations. Visual detection of the artificially introduced changes was possible by comparing the PG to the depth-dose profiles. Moreover, $2 \mathrm{~mm}$ range shifts could be detected in the head irradiation case using a simple linear regression fit to the falloff of the PG-profile. The influence of changes in complex, patient-like dose distributions on the PG-profiles obtained with multi-slat collimation is first studied in this work, which further gives insight on collimator design optimization and highlights its potential and simplicity for detecting proton treatment deviations over a wide range of Bragg peak positions.
\end{abstract}

\section{Introduction}

Proton therapy is a form of radiotherapy (RT) that uses proton beams to destroy solid tumors. The potential of proton RT over conventional (photon) RT is that it can deliver a high dose to the tumor with less overall dose to healthy-tissues, a factor very important to reduce the side effects of irradiation. This high dose conformity is enabled by the fact that protons release a large amount of their energy in a highly localized region, the Bragg peak (BP), just before they stop [1]. This makes proton therapy well suited for treating e.g., deep-seated and pediatric tumors.

Despite these physical advantages, treatment plans in proton therapy are often sub-optimal in clinical practice due to the relatively large uncertainty in the particle range in vivo [2]. Particle range strongly depends on tissue composition, density, and heterogeneities. The main causes of range uncertainty are related to: (1) the conversion of X-ray computed tomography (CT) data to proton interaction data [3]; and (2) patient morphological/anatomical and/or physiological changes occurring during the course of the therapy. Such changes may include, e.g., edema of the irradiated region [4], tumor regression [5], and filling of empty cavities due to inflammation and/or increased tissue permeability leading to edema.

Prompt-gamma (PG) imaging has been suggested for in vivo monitoring of proton therapy delivery, range verification in particular $[6,7]$.

\footnotetext{
* Corresponding author.

E-mail address: crespo@lip.pt (P. Crespo).
} 
This technique is based on the detection of gamma quanta originating from proton-nuclear interactions within the body, which are emitted promptly and can escape from the patient. Compared to PET imaging of $511 \mathrm{keV}$ gamma rays resulting from positron-emission decay of protoninduced radioactive nuclides [5,8-11], PG imaging does not rely on delayed emission and therefore is unaffected by biological processes (e.g., activity washout). Moreover, it may provide real-time feedback on the treatment delivery, on a pencil-beam basis.

PG rays are single photons, therefore Compton cameras [12-15], mechanically collimated cameras, PG spectroscopy [16], and timing spectroscopy [17] are currently being investigated to resolve spatial information. The scope of this work is mechanical collimation, which has the advantages of relative simplicity of implementation and compactness, as well as the possibility to provide complementary information beyond the particle range. Prompt-gamma profiles can be obtained by simple projection of the object onto the image plane, without Compton reconstruction. Moreover, full-energy deposition is not required, unlike in spectroscopy measurements where only few energy-resolved lines are usually targeted [16,18-22]. Kelleter et al. [21] present an experimental spectroscopy study. However, no variation in the distal fall-off of the Bragg peak is exploited. Zarifi et al. [22] present a Geant4-based Monte Carlo study with sub-millimeter range resolving power. However, the influence of a detection system (diminishing the counting statistics) is not taken into account, together with a validation of the prompt gamma yield, which has been shown to vary between the Geant4 Monte Carlo code in respect to the MCNPx and Fluka packages [23].

In pencil-beam scanning (PBS), the lateral position of the proton pencil beam is well known already, so obtaining information on the dose distribution along the beam direction is more important. Different types of collimators have been investigated, such as the multi-slat or multi-slit collimator [24-26], the pinhole collimator [27], and a onedimensional pinhole denoted as knife-edge slit [28-30]. Multi-slat collimators are made of multiple plates ('slats') made of a high-density material such as tungsten. The slats are typically placed perpendicular to the pencil-beam direction, although tapered configurations can also be used [31]. Previous studies have shown that collimated PG profiles correlate well with the distal dose falloff, both in simulations [23,29] and experiments $[25,28-30,32,33]$. Although the results are encouraging, the aforementioned works are proof-of-principle studies focused mostly on comparing collimated PG profiles to pristine Bragg peak distributions in homogeneous phantoms and/or phantoms with simple artificial inserts of different materials. Smeets et al. [34] have considered a multi-slat camera operated without the shifting time-of-flight (TOF) method, which results in $(\mathrm{n}, \gamma)$ reactions that disturb the optimum profile distribution. In addition, they compared two cameras (knifeedge slit vs multi-slat) with a weight limit being imposed on both, which may not allow optimal multi-slat camera operation. Heterogeneities are also not considered in that study. The first in vivo results of PG imaging have been recently reported using a knife-edge slit collimator, obtained during brain irradiation with pencil beam scanning [35] and passive beam collimation [36]. Krimmer et al. [37] provide a thorough review of prompt-gamma-based range verification.

In this work, we focus on developing multi-slat collimators that accept photons emitted at right angles from the beam axis, for proton therapy verification on a per-pencil-beam basis, and considering clinical realistic scenarios. Potential advantages in regard to the knife-edge slit collimator in this case, are that the multi-slat collimator allows for simultaneous imaging of the whole proton path and not only the dose falloff, due to the constancy of its geometric performance throughout its field of view (FOV).

There is still little knowledge on the optimal properties of a multislat collimator for PG imaging. Although the potential of multi-slat PG imaging has been inferred to some extent from PG measurements using a single collimated detector scanned parallel to the beam axis [32,33], these studies give little insight into the signal-to-background ratio in a full-scale system. Min et al. [25] took a first step towards optimizing a multi-slat collimator based on Monte Carlo simulations, but considered only one fixed distance between the phantom and the collimator and did not apply TOF background discrimination (due to (n, $\gamma$ ) reactions).

We perform Monte Carlo (Geant4) simulations of the PG profiles registered by various multi-slat configurations, upon proton irradiations of an NCAT (NURBS-based cardiac-torso) anthropomorphic phantom [38]. We study the viability of using a multi-slat collimated gamma camera, from the clinical deviation in dose delivery until its detection in the imaging system. The alterations in dose delivered in an anthropomorphic phantom studied in this paper correspond to real questions that arose in clinical situations, which some of the authors have experienced on the German heavy ion pilot project (GSI Darmstadt) (despite being then in the context of carbon ions). For example, it was sometimes wondered whether an undershoot or an overshoot was occurring during fractionated irradiation in the brain (possibly due to repeated damage to brain vascularization). One possible mechanism responsible for inducing such question was the fact that the images were obtained by in-beam PET, where washout was thought to play a major role. Here, washout plays no effect; therefore, this paper presents for the first time the advantage of imaging prompt gammas that are washout-free, taking into account a human phantom.

The simulated PG profiles obtained with different multi-slat collimators are compared to determine which collimator performs best. Furthermore, the influence of simple signal-processing options such as TOF discrimination, energy selection, and median filtering are studied. The detector system was not simulated on purpose, in order to give emphasis on the effect of the collimator itself, without compromising the different results that would be obtained by choosing one or other type of detector. By using a digital anthropomorphic phantom connected to simulations with a multi-slat collimation system, this work tries to estimate whether such a system may be useful in real clinical scenarios.

\section{Methods}

In the following section we briefly describe how the multi-slat configurations for further study were selected. Section 2.2 subsequently describes the Monte Carlo simulation framework, Section 2.3 the NCAT case studies, and Section 2.4 the post-processing of the simulation data.

\subsection{Definition of multi-slat collimator configurations}

The optimum slat height, slat thickness, and slit aperture (Fig. 15, Appendix A) for multi-slat collimators at different distances from the beam axis, were determined as previously explained by Cambraia Lopes et al [39], using Eqs. (1) and (2), that give its effective point resolution, $R_{\text {eff }}^{\text {multi-slat }}$, and its effective $2 D$ efficiency, $g_{\text {eff }}^{\text {multi-slat }}$ at the beam axis:

$R_{\text {eff }}^{\text {multi-slat }} \approx d\left(l_{\text {eff }}+b\right) / l_{\text {eff }}, l_{\text {eff }}=l-2 / \mu$

$g_{\text {eff }}^{\text {multi-slat }} \approx d^{2} /\left[2 \pi l_{\text {eff }}(d+t)\right]$, with $t=[6 d / \mu] /[l-(3 / \mu)]$

where $l$ is the slat height, $d$ the slit aperture in the beam direction, $b$ the distance between the beam axis and the front surface of the multi-slat collimator, $t$ is the slat thickness, and $l_{\text {eff }}$ is the effective collimator height, used to account for septa penetration [40], and calculated based on the linear attenuation coefficient $\mu=7.9 \times 10^{-2} \mathrm{~mm}^{-1}$ for $5 \mathrm{MeV}$ photons in tungsten [41]. The derivation of the effective $2 D$ efficiency is shown in Appendix A.

Here we study collimators at distances between the beam axis and their front surface (b) of $15 \mathrm{~cm}, 25 \mathrm{~cm}$, and $35 \mathrm{~cm}$, which are compatible with typical treatment scenarios. The applicability of one or another distance in practice will depend on the irradiated site, angle of incidence, and the size of the patient. For e.g., for pelvis or thorax irradiations, the minimum achievable value of $b$ may be higher than for 
Table 1

Characteristics of the multi-slat collimators studied. The weight was calculated for a pelvic collimator with length of $40 \mathrm{~cm}$ in the direction of the beam axis and a slit length of $50 \mathrm{~cm}$ (according to Fig. 2-right). The collimator weight for the case of a head irradiation equals half the value given here. The geometric parameters $b, l, d$, and $t$ are illustrated in Fig. 15 (in Appendix A).

\begin{tabular}{llllll}
\hline $\begin{array}{l}\text { Multi-slat } \\
\text { collimator }\end{array}$ & $\begin{array}{l}\text { Collimator } \\
\text { front- } \\
\text { surface to } \\
\text { beam } \\
\text { distance, } b \\
(\mathrm{~cm})\end{array}$ & $\begin{array}{l}\text { Collimator } \\
\text { height, } l \\
(\mathrm{~cm})\end{array}$ & $\begin{array}{l}\text { Slit } \\
\text { aperture } \\
\text { diameter, } \\
d \text { (mm) }\end{array}$ & $\begin{array}{l}\text { Slat/septa } \\
\text { thickness, } t \\
(\mathrm{~mm})\end{array}$ & Weight $(\mathrm{kg})$ \\
& 15 & 12 & 2.7 & 2.5 & 226 \\
\hline A & 15 & 16 & 2.8 & 1.7 & 237 \\
B & 25 & 18 & 2.7 & 1.4 & 240 \\
C & 35 & 18 & 7.3 & 3.9 & 251 \\
D & 15 & 20 & 5.1 & 2.4 & 250 \\
E & 25 & 20 & 3.8 & 1.8 & 251 \\
F & 35 & & &
\end{tabular}

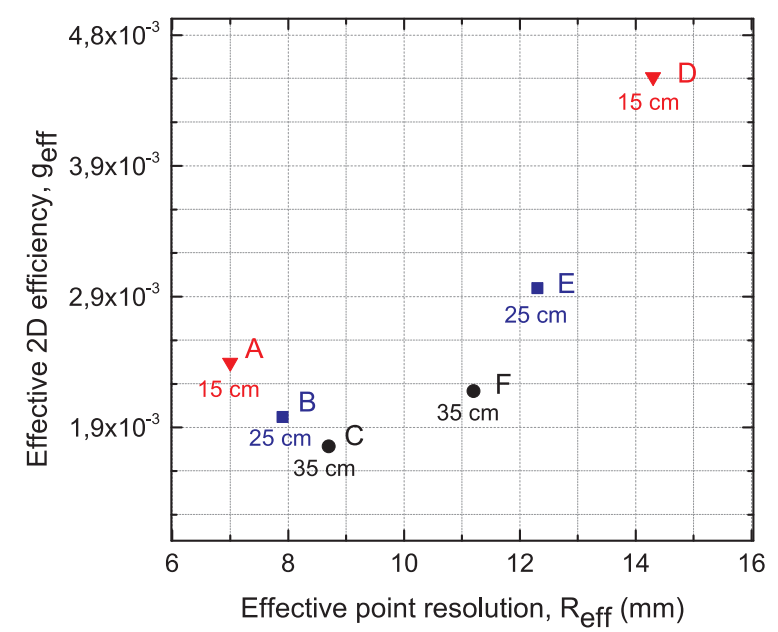

Fig. 1. Effective point resolution and 2D efficiency of the multi-slat collimators investigated. The source-to-collimator distances are specified in $\mathrm{cm}$.

head irradiations, especially in situations where oblique beam incidence is preferable. The geometrical properties of the six multi-slat collimators studied are listed in Table 1, and their corresponding performance characteristics are shown in Fig. 1. We used a slit length of $50 \mathrm{~cm}$, which corresponds to azimuthal angle coverage of the flat-panel detector, in the range $0.14-0.24$, depending on the collimator configuration. This slit length is considered a reasonable value for clinical application.

The method used to derive the optimal geometrical properties of the collimators [39] is general, i.e., by fixing any arbitrary desired collimator resolution (or efficiency) and distance from the beam axis, the geometrical parameters that give an optimal efficiency (or resolution) can be derived based on simple geometric considerations. For the purpose of the present work it is not important to reason the choice of the specific collimator resolutions or efficiencies used, but rather to compare collimators with different properties. Nevertheless, it is to be noted that for each distance from the beam, two optimal collimator geometries were used, one optimized for efficiency at a given fixed resolution (A-C), and the other optimized for resolution at a given fixed efficiency (D-F).

\subsection{Geant4 simulations}

Monte Carlo (MC) simulations were performed using the Geant4 toolkit $[42,43]$. The simulations were done in two steps, to reduce computation time. First, the low-energy electromagnetic physics package QGSP_BERT_HP of Geant4, version 9.3, was used to simulate the irradiation of an anthropomorphic phantom by a proton pencilbeam. The range cuts were set to $700 \mu \mathrm{m}$ for all particles. The absorbed dose in the phantom was stored in a cubic-millimeter grid covering the irradiated region. Furthermore, the properties of the particles escaping the phantom and crossing a surface fully containing the collimator field-of-view were registered in a phase-space file. The scored properties included the particle type, position, momentum, energy, and arrival time relative to the time of creation of the primary proton.

In a second step, the low-energy electromagnetic physics package QGSP_BERT_HP of Geant4 version 9.4 was used to simulate the propagation of the particles registered in the phase-space file through the collimator and towards an ideal flat-panel detector, located $1 \mathrm{~mm}$ behind the collimator exit. In this way, the phase-space file from each irradiation case study could be used to test multiple collimator geometries. Only particles escaping the phantom with a momentum higher than $100 \mathrm{eV} / \mathrm{c}$ were considered in these simulations, corresponding to a kinetic energy of $100 \mathrm{eV}$ for gammas and $5.34 \times 10^{-6} \mathrm{eV}$ for neutrons. The range cuts were set for all particles to $1 \mu \mathrm{m}$.

The particle type, position, momentum, energy, and time-of-flight were registered for each particle that hit the detector, post collimation. In addition, Compton scattering and the existence of a 'parent' particle (i.e. the detected particle is not the same as the one registered in the phase-space file) were tagged and stored for each particle detected. This information allowed distinguishing 'secondary' and 'direct' events, where direct events pertain to quanta that did not interact in the collimator. The time stamp (time-of-flight) of a detected particle was defined as the time difference between the launch of the proton and the arrival of the particle at the detector.

The anthropomorphic phantom was the NCAT phantom from Segars [38], which was previously implemented in Geant4 [44]. The incident proton energies were $130 \mathrm{MeV}$ for the head irradiations and $200 \mathrm{MeV}$ for the pelvis irradiation (no momentum spread). A single pencil-beam with a total of $10^{8}$ protons was simulated in each case study, which is representative of the most distal pencil-beam in a typical treatment plan [30]. The pencil-beam had a lateral full-width-at-half-maximum (FWHM) of $9 \mathrm{~mm}$, which is representative for presently-used PBS systems $[45,46]$.

The case studies were implemented as depicted in Fig. 2. The collimators were made of tungsten (density of $19.3 \mathrm{~g} \mathrm{~cm}^{-3}$ ). The total size of the collimator in the beam direction was $20 \mathrm{~cm}$ for the head irradiations and $40 \mathrm{~cm}$ for the pelvis case, so as to completely cover the irradiated region. The detector surface and collimator front- and backsurfaces were parallel to the phantom-axial plane behind the NCAThead (Fig. 2-left), or to the phantom-coronal plane above the NCATphantom pelvis (Fig. 2-right), respectively. Sustaining $250 \mathrm{~kg}$ (collimator weight in the prostate case) can be well accomplished with millimeter accuracy by using a robotic solution. Positioning the detector above the patient corresponds to imaging gamma rays that have escaped through more bone and biological tissue. Positioning the detector below the patient is also a feasible solution (not investigated here), although effects like the reduction of solid angle due to increased detector distance or scattering in the patient couch would have to be considered.

The collimators listed in Table 1 were used for the head irradiations. For the prostate case, only the collimators at distances of $b=25 \mathrm{~cm}$ and $b=35 \mathrm{~cm}$ were simulated.

To compare the secondary particle spectra obtained with the NCAT phantom to those obtained with a PMMA phantom, we additionally simulated a solid PMMA cylinder (20-cm diameter and 30- $\mathrm{cm}$ length; stoichiometry $\mathrm{C}_{5} \mathrm{H}_{8} \mathrm{O}_{2}$; density $1.18 \mathrm{~g} \mathrm{~cm}^{-3}$ ) irradiated by a $200 \mathrm{MeV}$ proton pencil beam.

Various studies have shown considerable overestimation of prompt gamma emission yields by Geant4, up to its version release 9.6, in comparison to other simulation codes $[23,47-50]$. Therefore, similar to a previous work [23], we adopted a conservative approach in which 

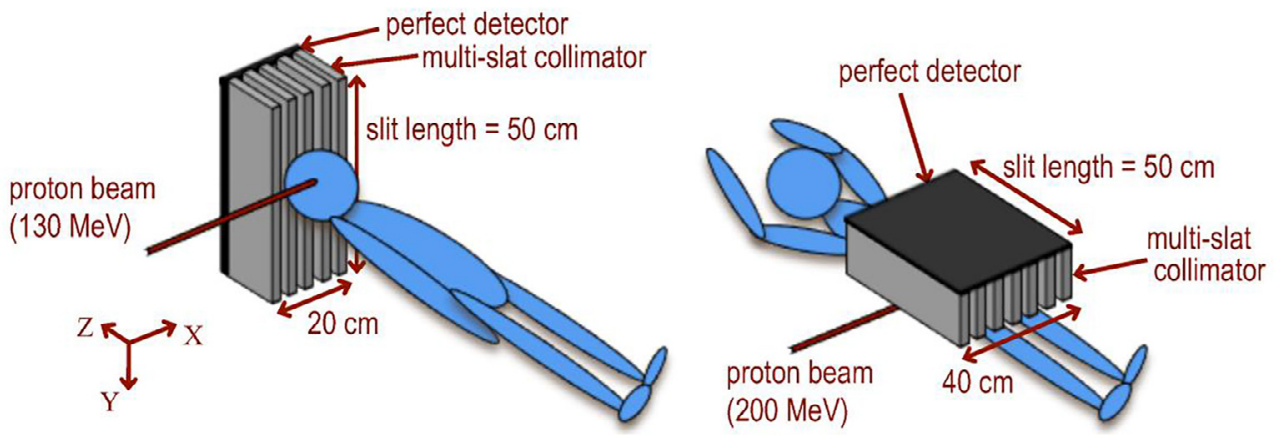

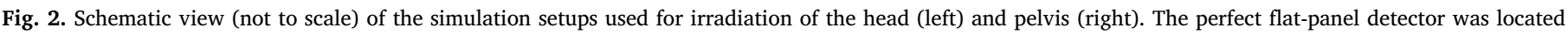
$1 \mathrm{~mm}$ behind the collimator.

only $25 \%$ of the total gammas escaping the NCAT phantom are tracked through the collimator. The gamma reduction factor of 4 thus applied followed from a comparison of the total gamma yields exiting a PMMA target ( $15 \mathrm{~cm}$ diameter and $20 \mathrm{~cm}$ length) obtained with our code, to that obtained by Smeets et al. [30] with MCNPX 2.5, under the same circumstances: proton energy equal to $160 \mathrm{MeV}$, perfect detection system, and no modeling of the room geometry. The average ratios of the gamma ( $1 \mathrm{MeV}-10 \mathrm{MeV})$ and neutron $(1 \mathrm{MeV}-160 \mathrm{MeV})$ counts found between Geant4 and MCNPX were 3.7 and 0.95, respectively.

\subsection{Case studies}

Three case studies were investigated: two head irradiations and one pelvis case. In each study, a reference ('planned') irradiation was compared to compromised irradiation(s), in which a phantom tissue property or set-up property was changed in a way that represents possible clinical inter-fractional changes with respect to the original treatment plan.

The first case study featured the irradiation of a head tumor located in the region of the sphenoid bone. A side irradiation was simulated with a horizontal (X direction in Fig. 2-left) pencil beam, which enters the temple of the head, crosses the nasal cavity, and stops at the distal sphenoid bone. In the planned irradiation, the nasal cavity was empty. In the compromised case, the nasal cavity was filled with water tissue (density $1.02 \mathrm{~g} \mathrm{~cm}^{-3}$ ). The compromised case could represent interfraction morphologic/physiologic changes such as the presence of mucus in response to a cold, edema or tissue swelling resulting from irradiation injury, or tumor growth.

The second case study consisted of an irradiation crossing the pituitary gland. A horizontal pencil beam also entered the head laterally (X direction in Fig. 2-left), but posterior to the first case, passing the sella turcica and the depression in the sphenoid bone that houses the pituitary gland. The difference between the compromised irradiations and the planned one is in the density of the brain tissue traversed by the protons (Fig. 10). The brain density in regions traversed by the beam was changed by $\pm 4 \%$. These changes represent possible responses to radiation injury: the hypo-dense case could result from vascular permeability and edema formation (with edema having 4\% less density then brain tissue). The hyper-dense scenario could arise from local protein congregation and other scar-tissue forming elements [51].

The third case study concerned the irradiation of a prostate tumor. The beam is horizontal and laterally incident (X-direction in Fig. 2right); the protons cross three pelvic bone structures before reaching the prostate. The proton energy required for this irradiation is among the highest used in clinical practice, thus constituting a worst-case scenario in terms of signal-to-background ratio [23]. The compromised irradiation was implemented by shifting the phantom $1 \mathrm{~cm}$ in the ventral (-Y) direction. Such a misalignment could e.g., result from a positioning/setup error or patient weight change.

\subsection{Post-processing of simulated data}

Post-processing of the simulated data included the shifting time-offlight technique and low-energy discrimination for background rejection. The aforementioned shifting TOF selection is based on a temporal shifting acceptance window, with 1 ns width and centered on the prompt-gamma peak, following the method described by Biegun et al. [23]. In addition, we have also calculated the impact of a cyclotron with a repetition rate of $100 \mathrm{MHz}$ (standard to worst case scenario with typical clinical cyclotrons), together with a proton bunch width of $1 \mathrm{~ns}$ FWHM. In case of synchrotron-based facilities it remains an open question whether or not TOF discrimination is applicable at all. The gamma profiles obtained under these circumstances are labeled "RR, $\Delta \mathrm{TOF}=1.5 \mathrm{~ns}$ ", meaning that the TOF window was opened by $1.5 \mathrm{~ns}$.

Subsequently, a median filter was applied to the registered gamma profiles to mitigate the periodic oscillations caused by the septa shadows, due to the proximity $(1 \mathrm{~mm})$ between the detector panel and the collimator back surface. Median filtering was implemented such that the median value of all data points lying within a given kernel width (span) was assigned to the point at the center of the kernel. In general, filtering the gamma profiles with a span covering at least two times the collimator pitch (i.e. the slit aperture plus slat thickness) was sufficient. However, for the profiles with the highest statistical fluctuations, a span of about four times the collimator pitch gave better results. In all cases, the span was kept as low as possible to avoid unnecessary degradation of resolution.

\section{Results and discussion}

\subsection{Energy and time spectra post-collimation: photon signal-to-background} ratio

The simulated time spectra of events scored at the perfect detector located just behind multi-slat E are shown in Fig. 3, for the NCAT head (left) and pelvis (right) irradiations. Photons that have interacted and/ or were created in the collimator are denoted as 'background.' Otherwise, they are denoted as 'direct'. The time spectra show that the direct photons arrive at the detector earlier than the neutrons, as expected due to that neutrons have mass. Since the time spectra were integrated over the entire detector area, the range of direct-photon time-of-flights is determined by the time needed by the protons to slow down inside the phantom, which is larger for $200 \mathrm{MeV}$ protons than for $130 \mathrm{MeV}$ protons. For this reason, there is a larger overlap between the directphoton and neutron time spectra in the pelvis irradiation case.

The time spectra of the secondary photons (Fig. 3, red) exhibit a local maximum within the same time region as the direct photons (i.e. between about $2 \mathrm{~ns}$ and $4 \mathrm{~ns}$ ). These events are attributed to secondary photons resulting from Compton interactions and/or electromagnetic cascades ('showers') initiated by the prompt gammas in the collimator channel collecting the primary photons. The remaining events have a 

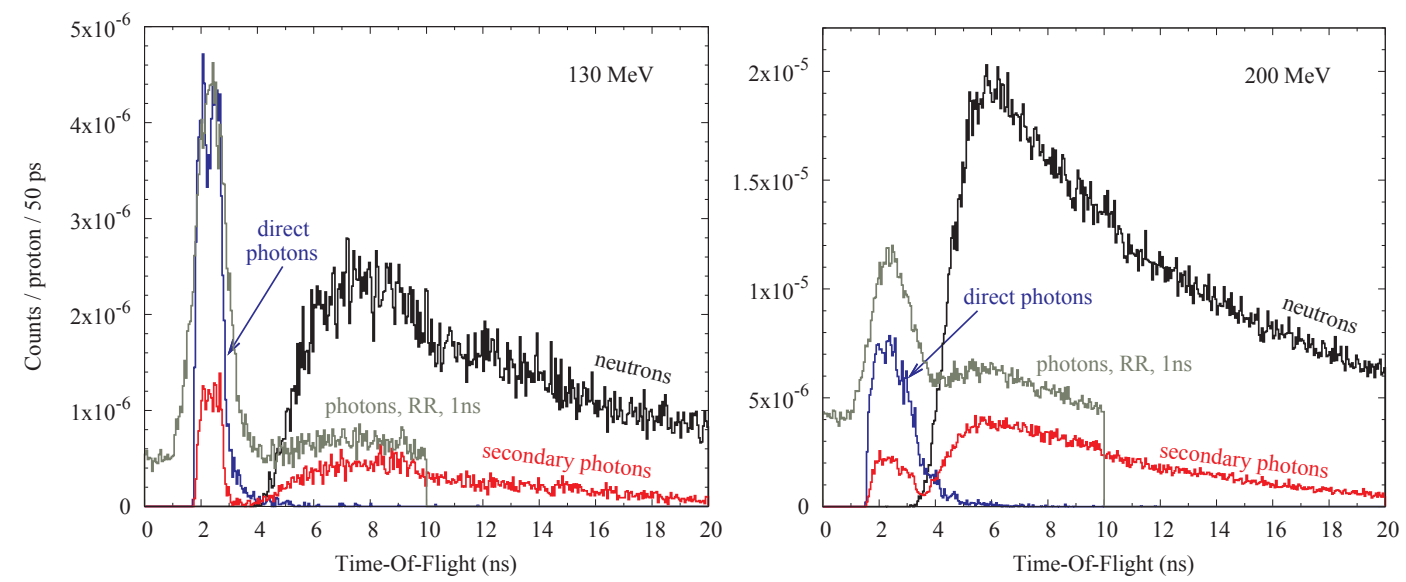

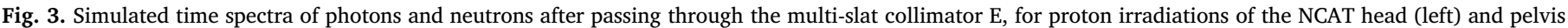

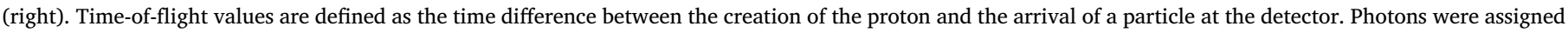

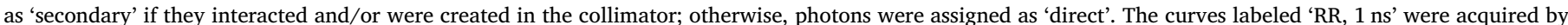

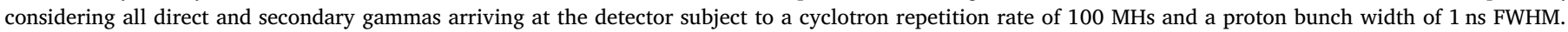

distribution of arrival times similar to that of the neutrons. These nonprompt background counts therefore are attributed to photons that are created in the collimator by neutron interactions.

The time spectra indicate that an improvement in the signal-tobackground ratio should be possible by accepting counts within the time window in which the direct photons are detected only. Indeed, it has been shown that such time-of-flight background rejection can be quite effective if the uncertainty in the proton arrival time (i.e. proton bunch width) is of the order of a nanosecond [20,23,31-33,52].

In Fig. 3, it can be seen that the direct-photon yield is a factor of about 2 higher for the pelvis irradiation than for the head case. However, the neutron yield of the $200 \mathrm{MeV}$ proton beam (pelvis) is about an order of magnitude higher than that of the $130 \mathrm{MeV}$ proton beam (head). Since the neutron-induced background gamma yield is directly proportional to the neutron yield, this implies that the signal-to-background ratio will be considerable worse in irradiations of pelvic, deepseated tumors. The curves labeled 'RR, 1 ns' in Fig. 3 were acquired by considering all direct and secondary gammas arriving at the detector subject to a cyclotron repetition rate of $100 \mathrm{MHz}$ and a proton bunch width of 1 ns FWHM.

We then investigate the ratio of direct-to-secondary photon counts, or photon signal-to-background ratio, $\mathrm{S} \gamma / \mathrm{B} \gamma$, for various combinations of low-energy cut and TOF acceptance window. We assume that the detector behind the collimator can be made essentially insensitive to neutrons (e.g., by using a scintillator with low neutron cross section). The results are shown in Fig. 4 for irradiations of the brain and the prostate. It is noted that phantom scatter is not discriminated from either signal or background since only interactions in the collimator were tracked.

For all multi-slat configurations, the $S \gamma / \mathrm{B} \gamma$ improves if the low-energy threshold is increased from $1 \mathrm{MeV}$ to $3 \mathrm{MeV}$, namely by a factor of 2.7-2.9 for the $130 \mathrm{MeV}$ proton beam and by a factor of 2.2-2.4 for the $200 \mathrm{MeV}$ beam. Moreover, the $\mathrm{S} \gamma / \mathrm{B} \gamma$ increases by an order of magnitude if TOF discrimination is applied in combination with a low-energy threshold of $1 \mathrm{MeV}$, compared to a $1 \mathrm{MeV}$ energy threshold only. Specifically, TOF discrimination improves the $S \gamma / \mathrm{B} \gamma$ by a factor of 7-9 for $130 \mathrm{MeV}$ protons and by a factor of $12-17$ for $200 \mathrm{MeV}$ protons. These results clearly favor the use of TOF discrimination in promptgamma imaging with multi-slat collimators. The same applies even if a repetition rate of a $100 \mathrm{MHz}$ is taken into consideration (standard to worst case scenario).

We furthermore observe that the $S \gamma / B \gamma$ increases from multi-slat configuration A up to E, regardless of the energy and TOF selection criteria applied. Multi-slat $\mathrm{F}$ has better $\mathrm{S} \gamma / \mathrm{B} \gamma$ than multi-slat $\mathrm{E}$ for the

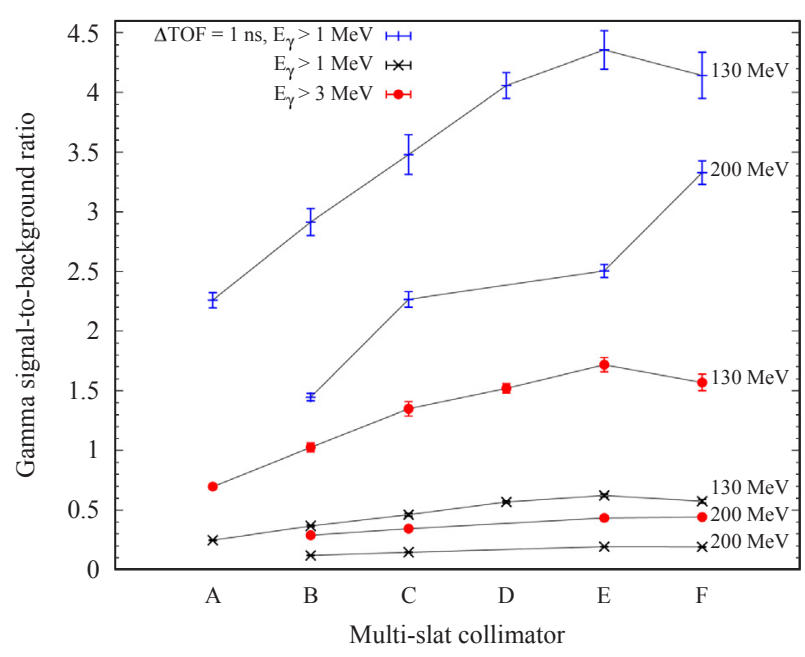

Fig. 4. Gamma signal-to-background ratios simulated for different energy (E $\gamma$ ) discriminations and for energy and TOF discrimination, for each of the multislat collimators studied (A-F), and for different proton energies: $130 \mathrm{MeV}$ protons (brain irradiation) and $200 \mathrm{MeV}$ (prostate irradiation). The signal is defined as the number of prompt gammas that are detected without interacting in the collimator. The background represents the remaining number of gammas detected. The lines are present to aid visualization. The beam microstructure was not taken into account in this analysis.

case of prostate irradiation with energy and TOF discrimination, while the results are similar to those of multi-slat $\mathrm{E}$ in the other cases. Fig. 1left, shows that the efficiency of multi-slat $A$ is better than that of $\mathrm{B}$, which in turn is better than that of $C$. The fact that the $S \gamma / B \gamma$ improves in the opposite direction is attributed to the increasing distance to the beam, which results in a decreasing exposure of the collimator to neutrons and non-perpendicularly incident photons.

This is confirmed by Fig. 5-left, which shows the energy spectra of multi-slat collimators A and C. All particle yields are higher for multislat A than for C. However, the difference in background (and neutron) levels is considerable higher than the difference in direct-photons counts, even when TOF discrimination is applied, and therefore $C$ has the best $S \gamma / B \gamma$. Similar arguments apply for comparing multi-slats D, E, and $\mathrm{F}$ : the relative decrease in direct-photon counts as a result of a decrease of efficiency from D to F (Fig. 1-left) is accompanied by a decrease in background due to a higher distance from the beam, therefore the $\mathrm{S} \gamma / \mathrm{B} \gamma$ increases only slightly for multi-slat $\mathrm{E}$.

Finally, when comparing multi-slat collimators with different 

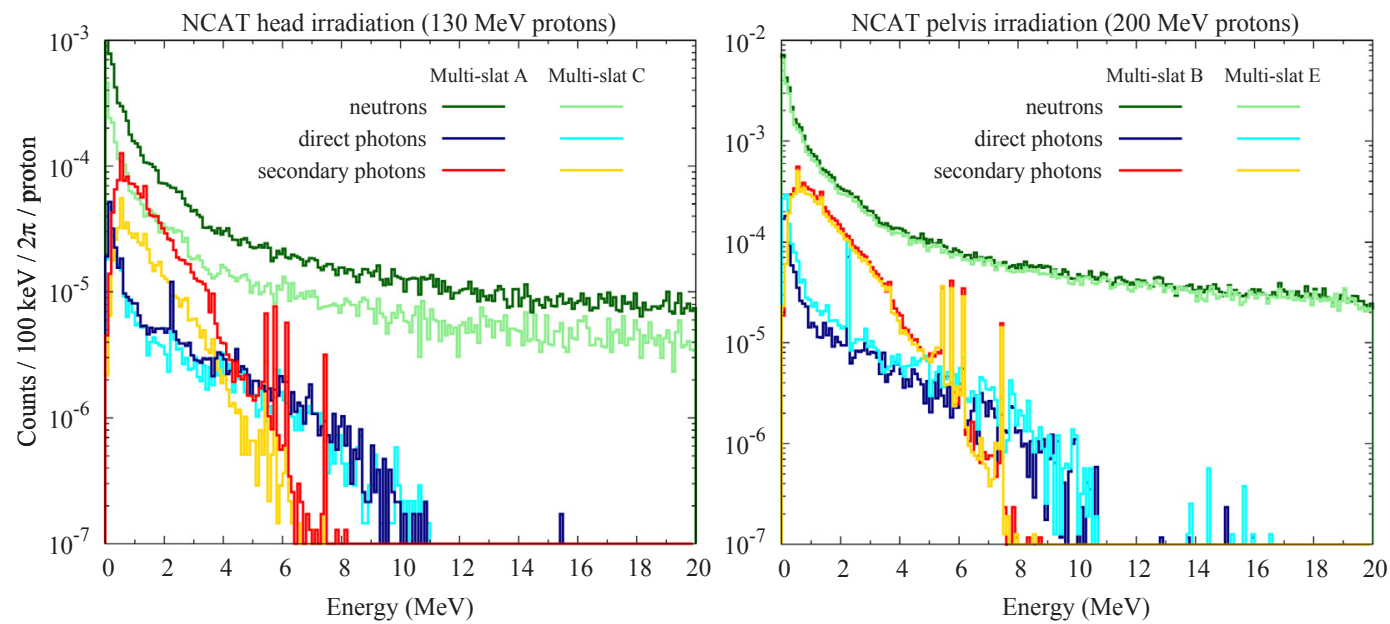

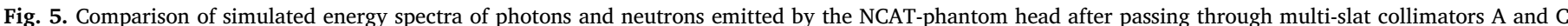

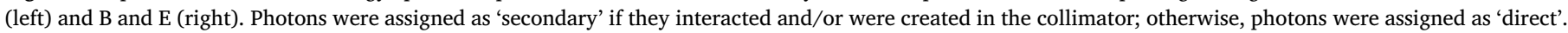
For comparison purposes, the counts were normalized with respect to the beam-azimuthal angle of the collimators.
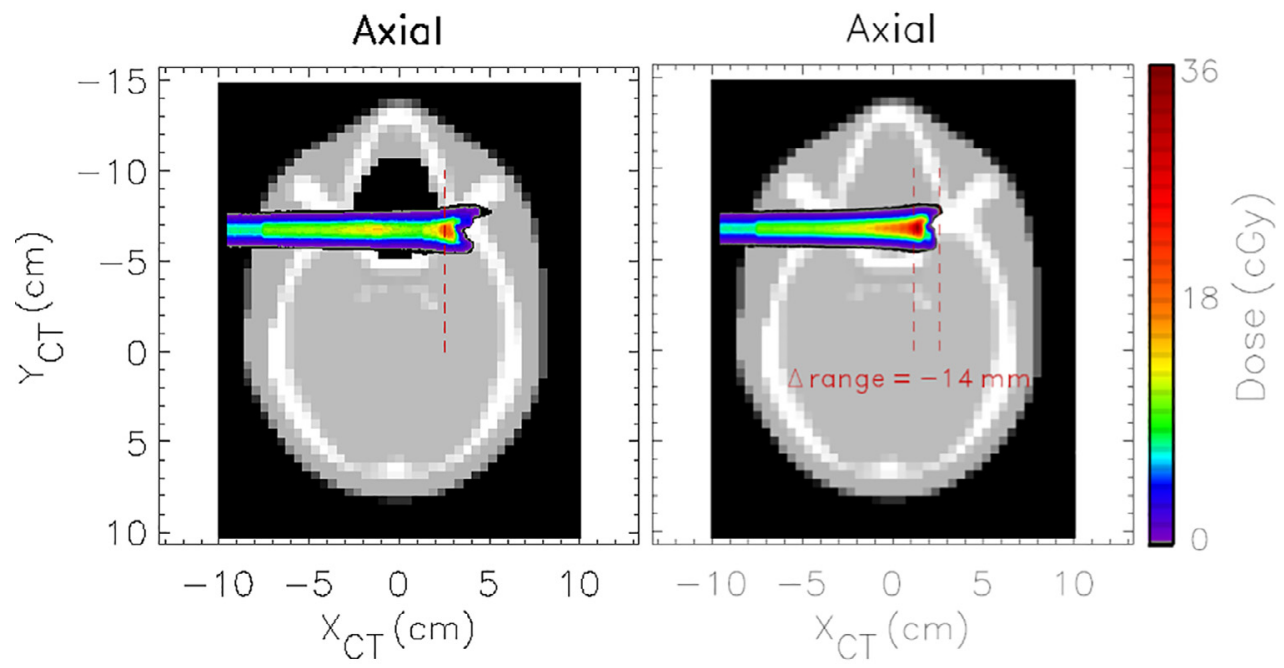

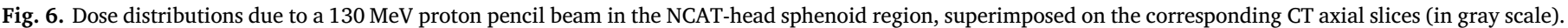
The proton range is reduced by $14 \mathrm{~mm}$ in the case of a filled nasal cavity (right) in comparison to the (planned) situation with an empty nasal cavity (left).

geometric performances placed at the same distance from the beam, as exemplified in Fig. 5-right for multi-slats B and E, we observe that the background levels are comparable, regardless of their resolution/sensitivity, as they emanate from the collimator at arbitrary directions. However, the higher efficiency of multi-slat $\mathrm{E}$ improves its $\mathrm{S} \gamma / \mathrm{B} \gamma$ compared to $\mathrm{B}$, by increasing the amount of direct photons, at the expense of resolution.

\subsection{Comparison between dose and collimated $P G$ profiles}

In this section, the collimated gamma profiles simulated with the various multi-slat configurations are visually compared with each other, and in regard to the corresponding dose distributions. For each of the three NCAT case studies, the PG profiles of a 'compromised' treatment scenario are presented comparatively to those of the 'planned' reference situation.

In all cases using TOF discrimination, two scenarios were considered: (1) a single-shot irradiation without overlapping consecutive bunches (e.g. as planned in laser-driven ion acceleration, [53]); and (2) irradiation with a cyclotron operating with a repetition rate of $100 \mathrm{MHz}$ (standard to worst case scenario) and a proton bunch width of $1 \mathrm{~ns}$ FWHM. In the first case a $\Delta$ TOF window width of 1 ns was used, whereas in the second case $1.5 \mathrm{~ns}$ width was applied.

The combinations with $\mathrm{E}>3 \mathrm{MeV}$ and $\Delta \mathrm{TOF}=1.5 \mathrm{~ns}$, with a repetition rate of $100 \mathrm{MHz}$ and a bunch width of $1 \mathrm{~ns}$ FWHM, were analyzed. These analyses were made both for the prostate irradiation as well as the head irradiation (not shown). In all cases, it was possible to observe that the sharpness of the distal edge diminished in the case of $\mathrm{E}>3 \mathrm{MeV}$. For this reason, all graphics with $\Delta \mathrm{TOF}$ analysis report to the case of $\mathrm{E}>1 \mathrm{MeV}$.

Based on the results in Section 3.3, already a pre-selection of the multi-slat collimators used to obtain the PG profiles can be done. As such, in the following sections we will restrict the results to the profiles obtained with TOF discrimination with the exception of one illustrative multi-slat collimator for which also the profile with only energy discrimination is shown. Although multi-slat collimators A-C show relatively poor gamma signal-to-background values than the others, in the following section it is interesting to compare the profiles of at least two of these cases, in order to show that improved collimator resolution and efficiency (e.g. A vs C) does not necessarily give an advantage if the background level is higher (e.g. smaller distance to beam).

\subsubsection{Irradiation of the sphenoid region: morphological changes}

The 2D dose distributions resulting from the irradiations of the 

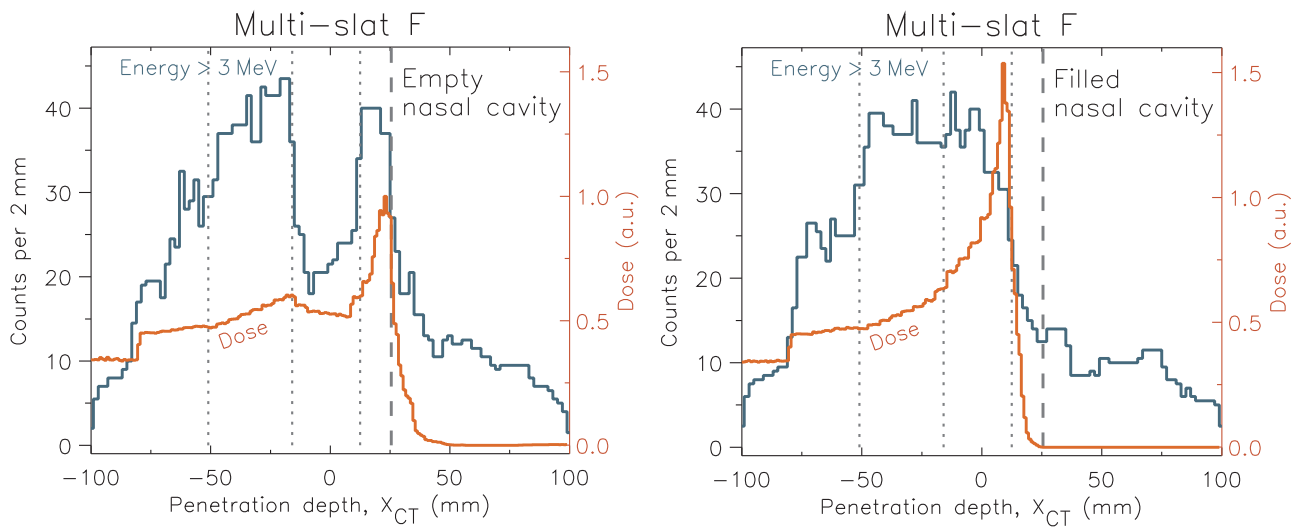

Fig. 7. Collimated prompt gamma profiles simulated for the irradiation of the NCAT sphenoid region (Fig. 6), using the multi-slat collimator F, and a low-energy threshold of $3 \mathrm{MeV}$. Also shown are the laterally-integrated depth-dose profiles. A median filter with a span of 6 bins was applied (bin size $=2 \mathrm{~mm}$ ). The vertical dashed lines correspond to the proton range in the planned, 'Empty nasal cavity' situation. The vertical dotted lines represent the softtissue/bone, bone/nasal-cavity, and nasalcavity/bone interfaces traversed by the protons, from left to right, respectively. sphenoid region are presented in Fig. 6, superimposed on a CT axialslice of the phantom head. We observe that the filling of the nasal cavity causes the pencil beam to stop $14 \mathrm{~mm}$ proximal with respect to the treatment plan, which took into account an empty nasal cavity. This under-range implies considerable underdosage in the tumor located in the sphenoid bone, with the distal part of the tumor not receiving any dose from this spot irradiation.

The collimated PG profiles obtained with only energy selection (low energy threshold of $3 \mathrm{MeV}$ ) are shown in Fig. 7 for multi-slat F, due to its highest gamma signal-to-background ratio $(\mathrm{S} \gamma / \mathrm{B} \gamma)$ and good resolution. The profiles obtained with a low-energy threshold of $1 \mathrm{MeV}$ and TOF discrimination are presented in Figs. 8 and 9. The vertical dotted lines in these figures indicate the successive interfaces traversed by the protons in the 'empty nasal cavity' situation, i.e. soft-tissue/bone $\left(\mathrm{X}_{\mathrm{CT}}=-5.1 \mathrm{~cm}\right)$, bone/nasal-cavity $\left(\mathrm{X}_{\mathrm{CT}}=-1.6 \mathrm{~cm}\right)$, and nasalcavity/bone $\left(\mathrm{X}_{\mathrm{CT}}=1.2 \mathrm{~cm}\right)$. Of course, these interfaces are not step-like but irregular across the beam diameter; they are meant to guide visual comparison between different figures.

The longitudinal dose profiles are indicated by the red curves in Figs. 7-9. These profiles represent the integral of the 3D-dose distribution in the $\mathrm{Y}$ and $\mathrm{Z}$ directions for each $\mathrm{X}$ value (Fig. 2). The reference ('empty nasal cavity') BP position, defined as the position at
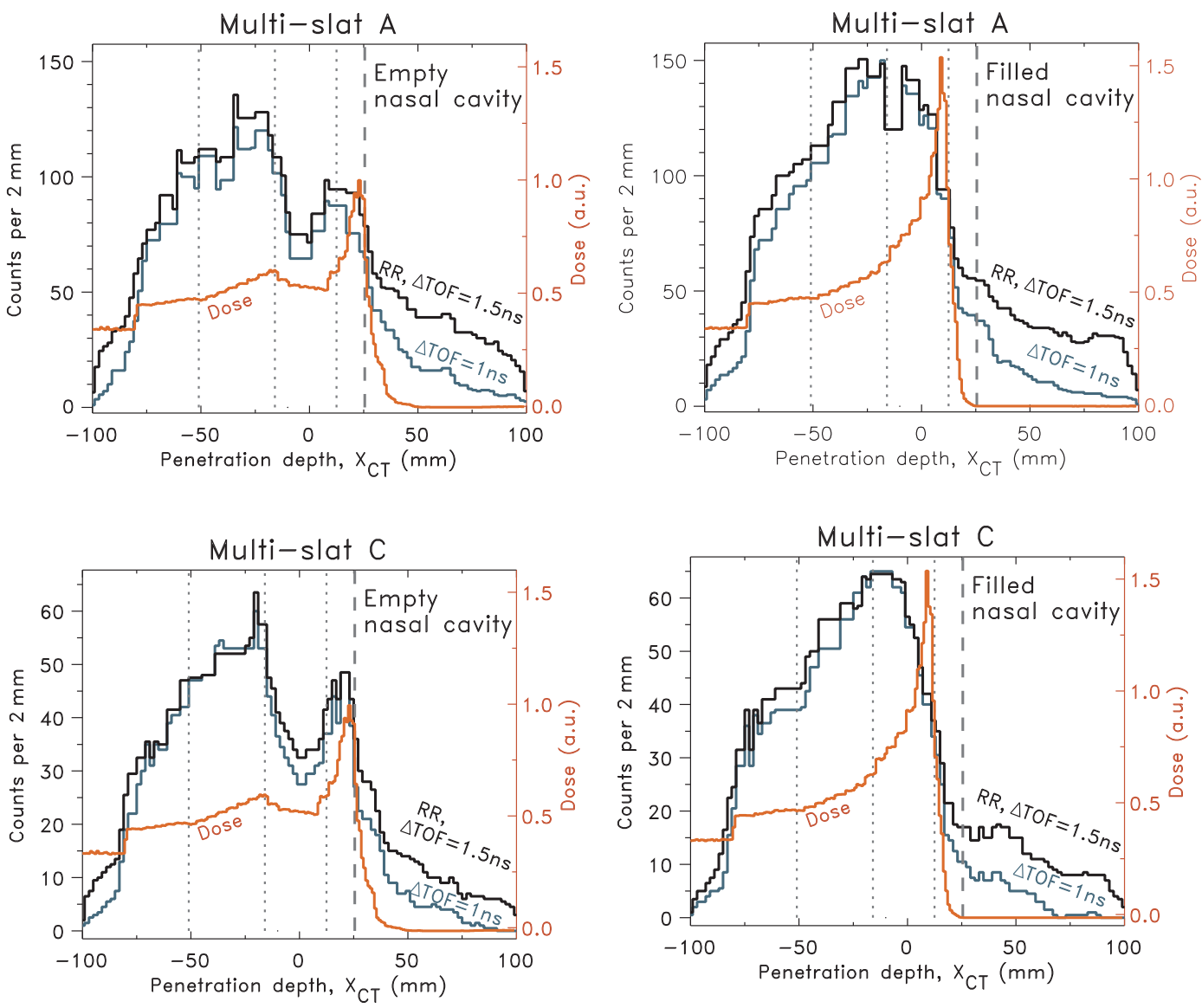

Fig. 8. Collimated prompt gamma profiles simulated for the irradiation of the NCAT sphenoid region (Fig. 6), using the multi-slat collimators A and C, and a lowenergy threshold of $1 \mathrm{MeV}$. Profiles are shown with ("RR, $\Delta \mathrm{TOF}=1.5 \mathrm{~ns}$ ") and without (" $\Delta \mathrm{TOF}=1 \mathrm{~ns}$ ") considering the beam microstructure. Also shown are the laterally-integrated depth-dose profiles. A median filter with a span of 8 bins was applied for both collimators (bin size $=2 \mathrm{~mm}$ ). The vertical dashed lines correspond to the proton range in the planned, 'Empty nasal cavity' situation. The vertical dotted lines represent the soft-tissue/bone, bone/nasal-cavity, and nasal-cavity/bone interfaces traversed by the protons, from left to right, respectively. 

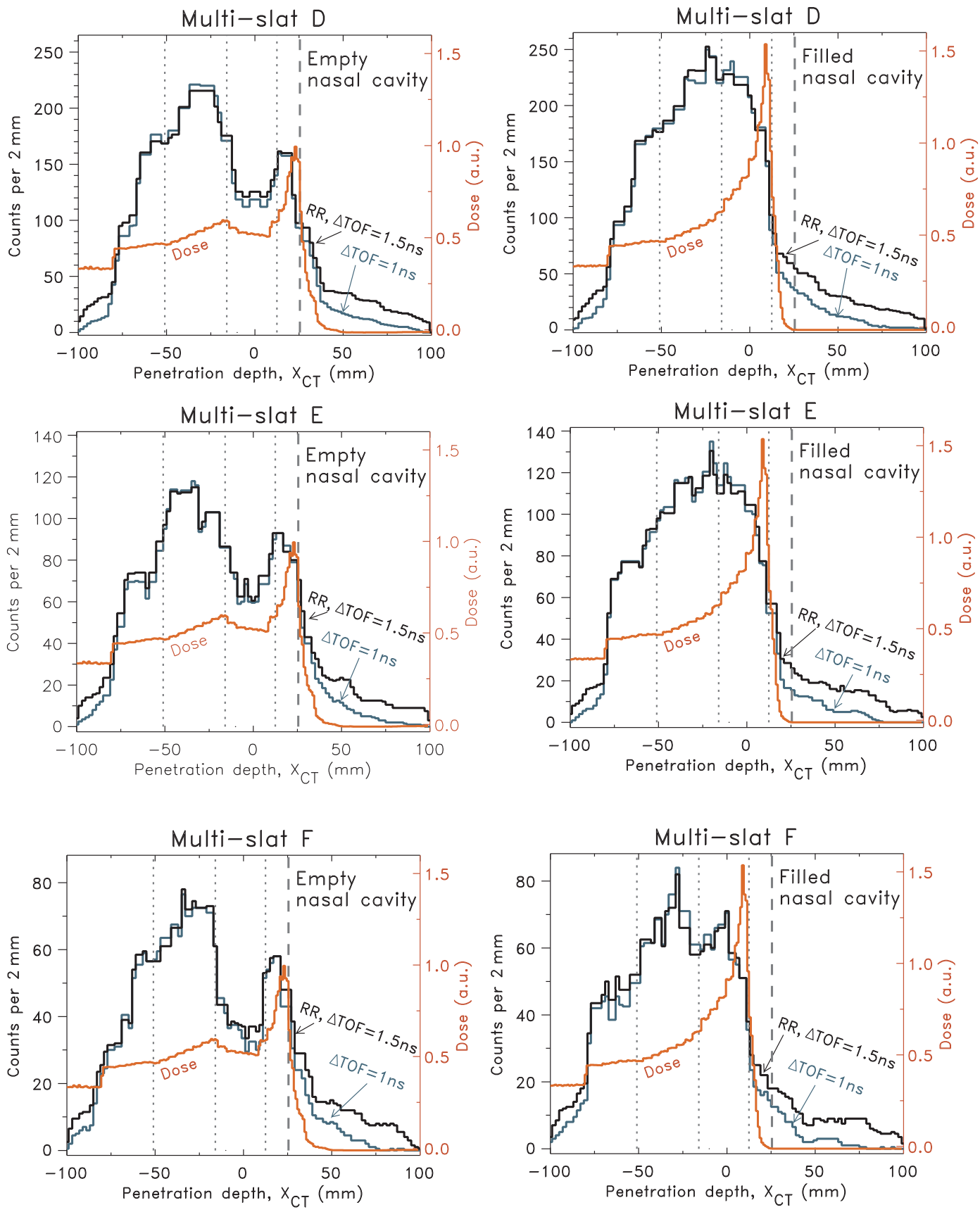

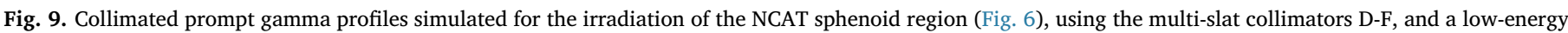

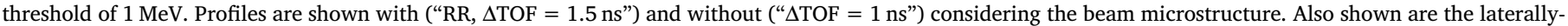

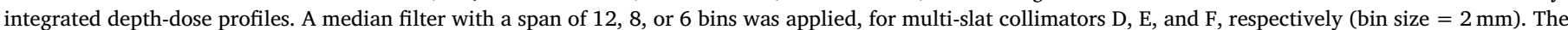

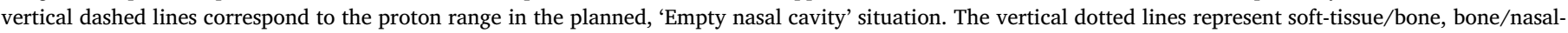
cavity, and nasal-cavity/bone interfaces traversed by the protons, from left to right, respectively.

which the dose has decreased to $80 \%$ of the maximum, is indicated by the dashed lines at $\mathrm{X}_{\mathrm{CT}}=2.55 \mathrm{~cm}$.

The profiles obtained with simultaneous energy and TOF discrimination present better counting statistics than the ones with only energy discrimination, due to the lower energy threshold of $1 \mathrm{MeV}$ that was possible to apply in the TOF-based profiles (Fig. 9-bottom vs Fig. 7). More importantly, the addition of TOF discrimination greatly increases $\mathrm{S} \gamma / \mathrm{B} \gamma$ and therefore enhances the correlation between the prompt gamma and depth-dose profiles. The profiles from multi-slat $\mathrm{E}$ and $\mathrm{F}$ in particular exhibit very sharp distal falloffs, which correspond to the depth-dose falloff within millimeter agreement. Furthermore, the patient entry position and the boundaries of the air cavity are clearly visible in the 'empty nasal cavity' case.

Finally, despite multi-slat A having the highest geometric resolution of all collimators studied, the spatial features in the profiles are visually less sharp when compared to multi-slats C-F, due to the higher background level of the first. This is especially noticeable at the boundaries of the air cavities and the bones, indicated by the dotted vertical lines, which correspond to transitions between tissues with very different densities. In particular, the air cavity is less clearly resolved for multislat A compared to multi-slat $\mathrm{E}$, while the overall number of counts are roughly the same and the geometric resolution of multi-slat A is considerably better. The effective resolution of the collimators seems therefore to be compromised due to secondary gammas (e.g., scattering 


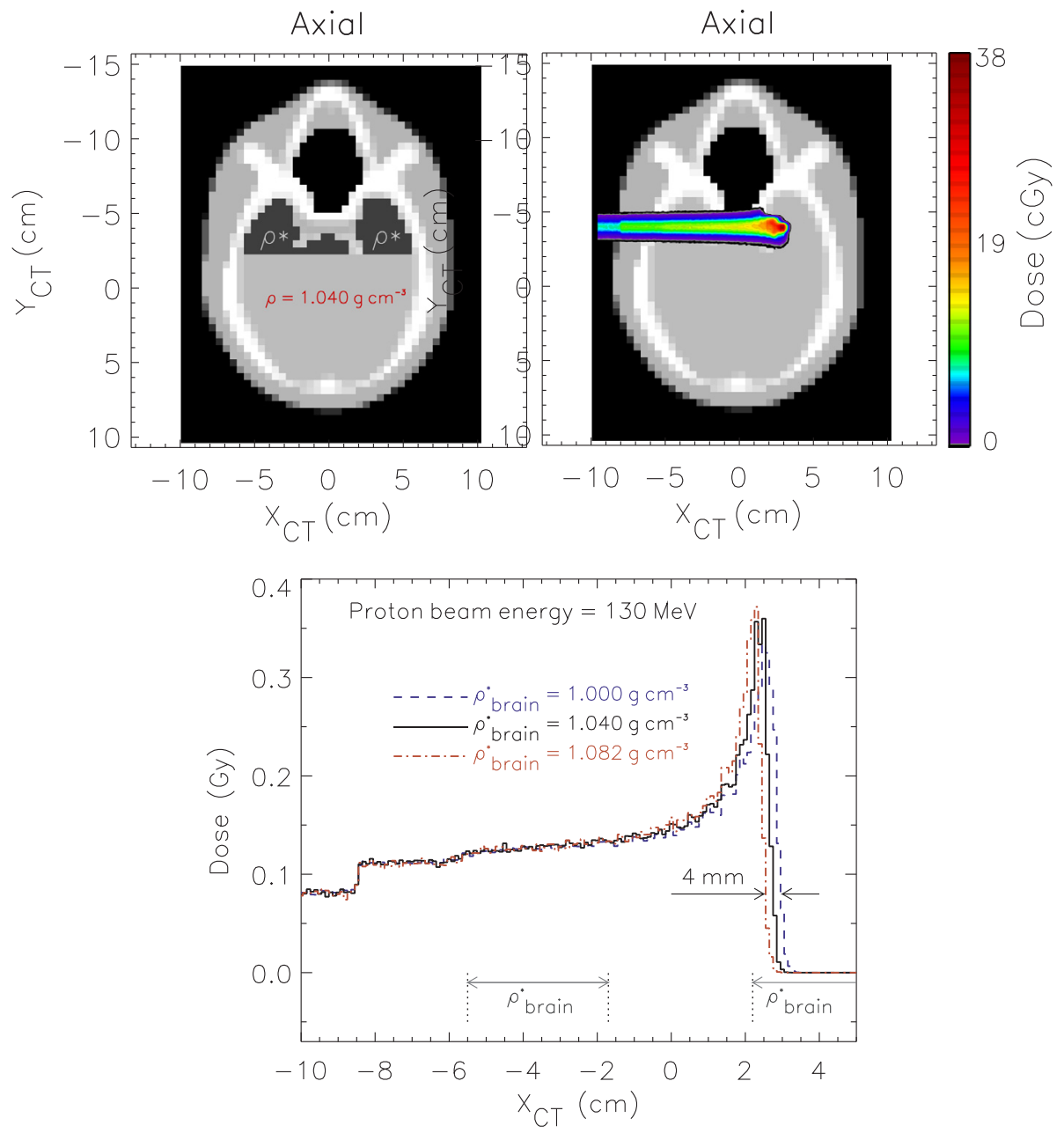

Fig. 10. Top-left: NCAT-head axial slice showing the region in dark-grey color where the brain density was changed ( $\rho$ ") by $\pm 4 \%$ relative to the original value $\left(\rho=1.040 \mathrm{~g} \mathrm{~cm}^{-3}\right)$. Top-right: Dose distribution corresponding to $130 \mathrm{MeV}$ proton irradiation of the pituitary gland (original brain density). Bottom: depth-dose profiles in the center of the pencil beam for the three brain-density cases studied.

in the collimator material (Section 3.3)), which are not rejected by TOF. To conclude, the choice between collimator $\mathrm{D}, \mathrm{E}$, and $\mathrm{F}$ will ultimately depend on the number of counts obtained in practice, and is thus a compromise between resolution and efficiency.

\subsubsection{Pituitary irradiation: small density changes}

The planned 2D dose distribution for the irradiation of the pituitary gland, under the reference condition of normal brain tissue density, is presented in Fig. 10-top-right, superimposed on a CT axial-slice of the phantom head. In Fig. 10-bottom, we show the longitudinal dose profiles obtained for the three brain densities simulated. The brain region where the density was changed to $\rho^{*}$ is colored dark-grey in Fig. 10-topleft. We observe that an increase or decrease in brain density by $4 \%$ results in range shifts of $-2 \mathrm{~mm}$ or $+2 \mathrm{~mm}$, respectively, in regard to the reference case (Fig. 10-bottom). Such range shifts may have clinical importance, especially when the BP is located inside the tumor or in the vicinity of an organ at risk.

To assess the capability to resolve these small range changes, we study the profiles obtained with the multi-slat configuration and background rejection that yielded the best $S \gamma / B \gamma$ ratio, i.e. with multislat $\mathrm{E}$ and simultaneous energy and TOF discrimination. These profiles are shown in Fig. 11-left, together with the normalized, longitudinal dose distribution in the reference situation. The range assessment was performed by a linear regression fit to the data points in the distal falloff region, between 30 counts and the 70 counts (Fig. 11-right). The range was estimated as the position in depth ( $\mathrm{X}_{\mathrm{CT}}$ direction) corresponding to the $50 \%\left(E_{50}\right)$ level of the fitted curves; i.e. $\mathrm{E}_{50}=\left(0.5 \times \max -p_{0}\right) / p_{1}$, where $p_{1}$ is the slope, $p_{0}$ is the offset, and max is the profile maximum.

These range estimations and their offsets in regard to the reference proton range are given in Table 2 . The reference proton range, for a normal brain density, is $\mathrm{R}_{70}=110 \mathrm{~mm}\left(\mathrm{X}_{\mathrm{CT}}=25.5 \mathrm{~mm}\right)$, and was defined as the phantom depth at which the dose has decreased to $70 \%$ of the depth-dose maximum. The $70 \%$ dose point was chosen instead of the usual $80 \%$ point for pencil beams, taking into account that the dose distribution presents lateral asymmetry (Fig. 10-top-right). This is because the protons traversing bone tissue stop before those traversing brain only.

As shown in Table 2, there is a clear difference between the $\mathrm{E}_{50}$ values obtained for the three density cases. The use of improved algorithms for range estimation, which are less sensitive to statistical oscillations in the gamma profile, are necessary to reduce the differences between the estimated and true proton range. Nevertheless, the simple method presented here already shows encouraging range-resolving capabilities. However, in addition to a sigmoid fit applied to the distal edge region (as in e.g. [52]) of the profile, the linear regression fit presented here could profit from user interaction, with the latter defining the start and stop positions of the fit, thus keeping simplicity of the method. Other statistical approaches have been described in the literature, e.g. [54], where the calculation of the range deviation is based on the minimization of the absolute profile differences in the distal part of two activity depth profiles shifted against each other. 

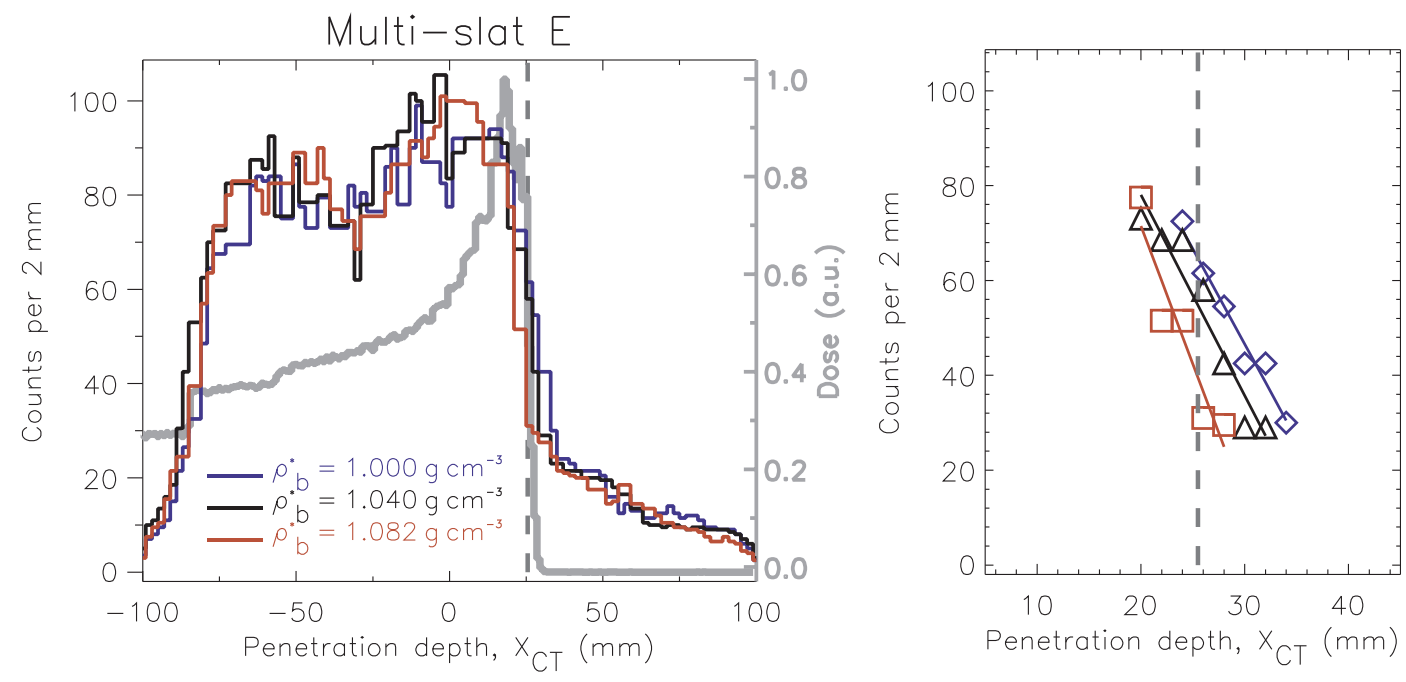

Fig. 11. Left: Collimated prompt gamma profiles simulated for the irradiation of the NCAT pituitary region (Fig. 10), using multi-slat collimator E. Also shown is the laterally-integrated depth-dose profile in the case of original brain density $\left(\rho_{b}^{*}=1.040 \mathrm{~g} \mathrm{~cm}^{-3}\right)$. A low-energy threshold of $1 \mathrm{MeV}$ and a TOF acceptance window of $1.5 \mathrm{~ns}$ were applied, together with a median filter with a span of 8 bins (bin size $=2 \mathrm{~mm}$ ). Profiles are shown considering the beam microstructure. Right: data points in the distal falloff region of each profile, between the 30 counts and the 70 counts of each profile, for over-dense (red squares), normal (black triangles), and hypodense brain tissue (blue diamonds), together with the corresponding linear regression fits. The vertical dashed lines in both graphs indicate the proton range in the case of the original brain density. (For interpretation of the references to color in this figure legend, the reader is referred to the web version of this article.)

\section{Table 2}

Range estimation corresponding to the $50 \%\left(\mathrm{E}_{50}\right)$ level of the gamma-profile maximum, using a linear regression fit to the distal falloff region between the 30 counts and the 70 counts of each profile maximum (Fig. 11). $\mathrm{X}_{\mathrm{CT}}$ corresponds to the estimated position of the Bragg peak in the coordinate system (Xaxis) of Fig. $11 . \mathrm{R}_{70}=110 \mathrm{~mm}$ is the range determined by the phantom depth at which the depth-dose profile of a normal density (planned) has decreased to $70 \%$ of the maximum. The range values corresponding to over- and hypo-brain density are $108 \mathrm{~mm}$ and $112 \mathrm{~mm}$, respectively. The uncertainties correspond to one standard deviation and were obtained from propagation of the uncertainty in the fit results and the statistical uncertainty of the profile maximum.

\begin{tabular}{clll}
\hline Brain density & $\mathrm{X}_{\mathrm{CT}}(\mathrm{mm})$ & $\begin{array}{l}\text { Range estimation } \mathrm{E}_{50} \\
(\mathrm{~mm})\end{array}$ & $\begin{array}{l}\text { Offset to planned range } \\
\mathrm{E}_{50}-\mathrm{R}_{70}(\mathrm{~mm})\end{array}$ \\
\hline $\begin{array}{c}\text { Over-dense } \\
(+4 \%)\end{array}$ & 23.6 & $108.1 \pm 7.0$ & -1.9 \\
$\begin{array}{l}\text { Normal } \\
\text { Hypo-dense }\end{array}$ & 26.0 & $110.5 \pm 4.8$ & 0.5 \\
$(-4 \%)$ & 29.3 & $113.8 \pm 3.9$ & 3.8 \\
\hline
\end{tabular}

\subsubsection{Prostate irradiation: patient setup error}

The 2D dose distributions obtained from the irradiation of the prostate are presented in Fig. 12. We observe that the misalignment of $1 \mathrm{~cm}$ (patient to ventral, Fig. 12-bottom) causes a dose displacement to proximal and, consequently, tumor underdosage, in regard to the planned situation. Some protons traverse less bone tissue, contributing to an excess range and not to the correct, expected range. The $80 \%$ dose point from the laterally-integrated depth-dose profile in the compromised scenario is shifted by $11.8 \mathrm{~mm}$ in the proximal direction, in comparison to the reference irradiation. The prostate is situated between the bladder and the rectum, both of which are organs-at-risk. It can also be seen that the misalignment in this example causes higher dose deposition in the lining of the rectum.

Collimated gamma profiles are shown in Figs. 13 and 14. As before, in all graphs there are vertical dotted lines to assist in the visualization of the successive interfaces traversed by the protons, in this case softtissue/bone $\left(\mathrm{X}_{\mathrm{CT}}=-14.5 \mathrm{~cm}\right)$, bone/soft-tissue $\left(\mathrm{X}_{\mathrm{CT}}=-4.6 \mathrm{~cm}\right)$, softtissue/prostate $\quad\left(\mathrm{X}_{\mathrm{CT}}=-2.4 \mathrm{~cm}\right), \quad$ and prostate/soft-tissue $\left(\mathrm{X}_{\mathrm{CT}}=1.5 \mathrm{~cm}\right)$. Again, these interfaces are not step-like, due to the tissue irregularities along the lateral span of the pencil beam. For instance, in Fig. 12-bottom, only about $1 / 3$ of the lateral pencil-beam

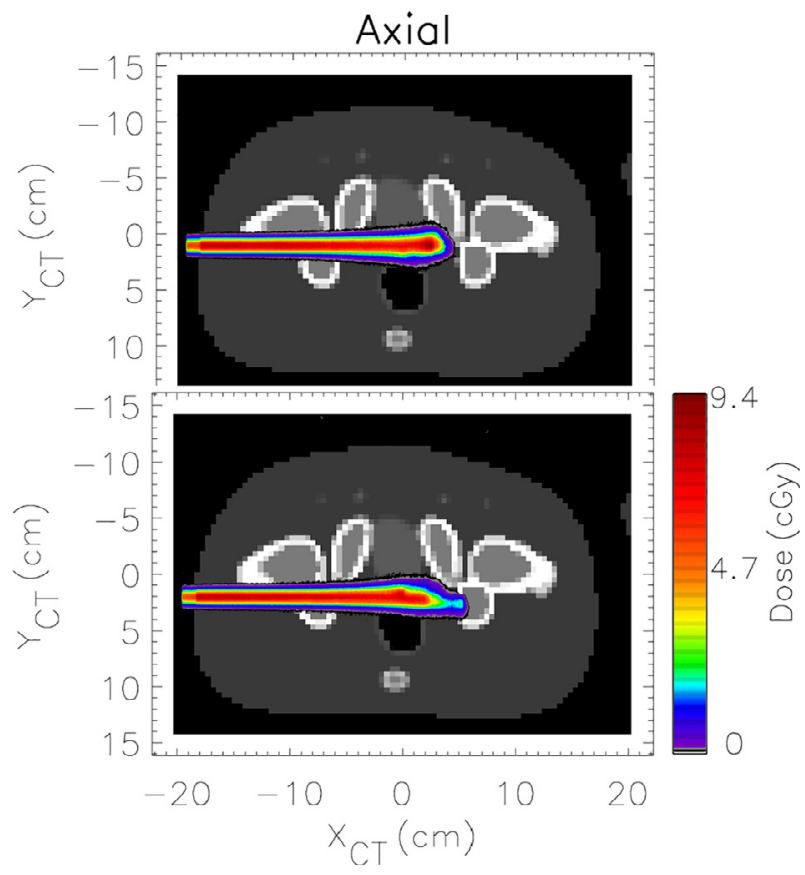

Fig. 12. Dose distributions for $200 \mathrm{MeV}$ proton pencil beam irradiation of the NCAT prostate region, superimposed on the corresponding CT axial slices (in gray scale, where windowing was done to improve visual contrast between soft tissues). Visual inspection reveals a proximal, longitudinal dose displacement, as well as tumor under-dosage in the case of a misalignment, where the patient is shifted $1 \mathrm{~cm}$ in the ventral direction (bottom), in comparison to the planned case (top).

span at the ventral-most side passes through bone tissue. In each graph, the red curves indicate the longitudinal dose profiles, integrated over the $\mathrm{Y}$ and $\mathrm{Z}$ directions. The reference ('planned treatment') BP position is indicated in all graphs by the dashed lines at $\mathrm{X}_{\mathrm{CT}}=2.8 \mathrm{~cm}$, and was defined as the traditionally used position at which the dose has decreased to $80 \%$ of its maximum.

The profiles obtained with energy discrimination only ( $3 \mathrm{MeV}$ lowenergy threshold, Fig. 13) are more difficult to interpret than those 

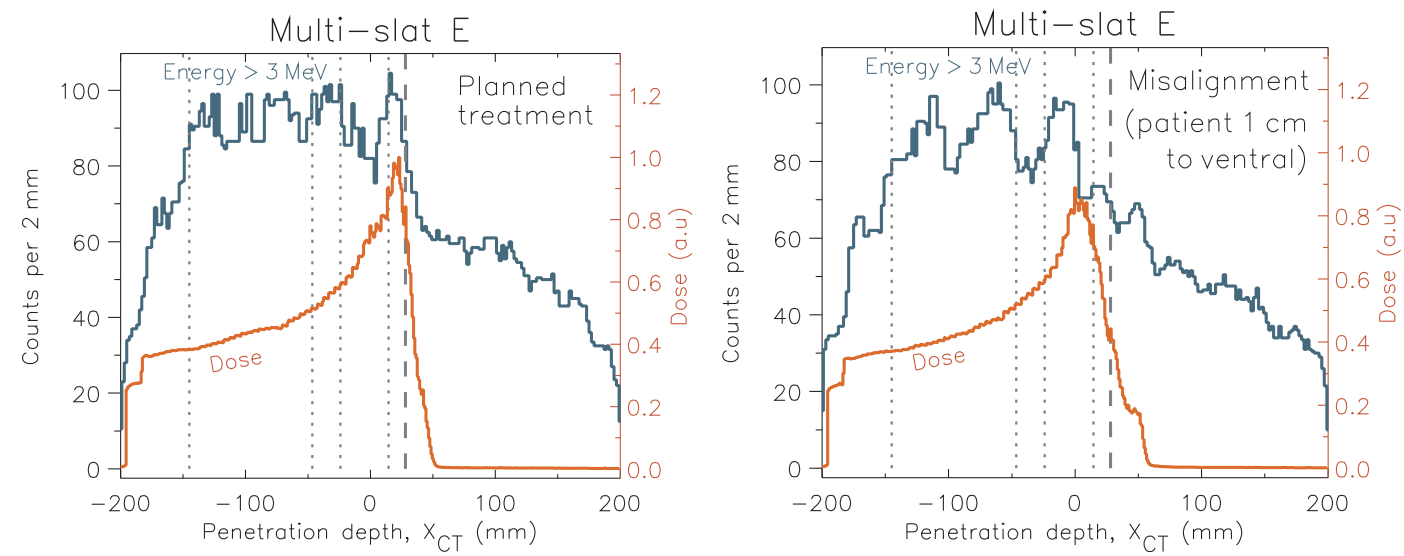

Fig. 13. Collimated prompt gamma profiles simulated for the irradiation of the NCAT prostate (Fig. 12), using multi-slat collimator E, and a low-energy threshold of $3 \mathrm{MeV}$. Also shown are the laterally-integrated depth-dose profiles. A median filter with a span of 8 bins was used (bin size $=2 \mathrm{~mm}$ ). The vertical dashed lines correspond to the proton range in the 'planned treatment' situation. The vertical dotted lines represent the soft-tissue/bone, bone/soft-tissue, soft-tissue/prostate, and prostate/soft-tissue interfaces traversed by the protons, from left to right, respectively.

obtained in the head irradiation cases because of the increased number of background gammas (Fig. 4). Not only do the more energetic protons generate more neutrons, also the collimator volume is twice as large as in the head irradiation cases, which may also contribute to higher amount of background. Therefore, the profiles with energy discrimination are only shown for one collimator (multi-slat E). While the falloff of the gamma profile from the 'planned treatment' case in Fig. 13 visually correlates with the corresponding depth-dose distal falloff, it is less trivial to retrieve the BP position from the gamma profile in the 'misalignment' case. In the latter profile 'bumps' are observable, which disturb the proper interpretation of the image, does leading to the necessity of applying the shifting TOF technique.

The gamma profiles obtained with simultaneous energy (1 MeV) and TOF discrimination (Fig. 14) present better $\mathrm{S} \gamma / \mathrm{B} \gamma$ than with energy
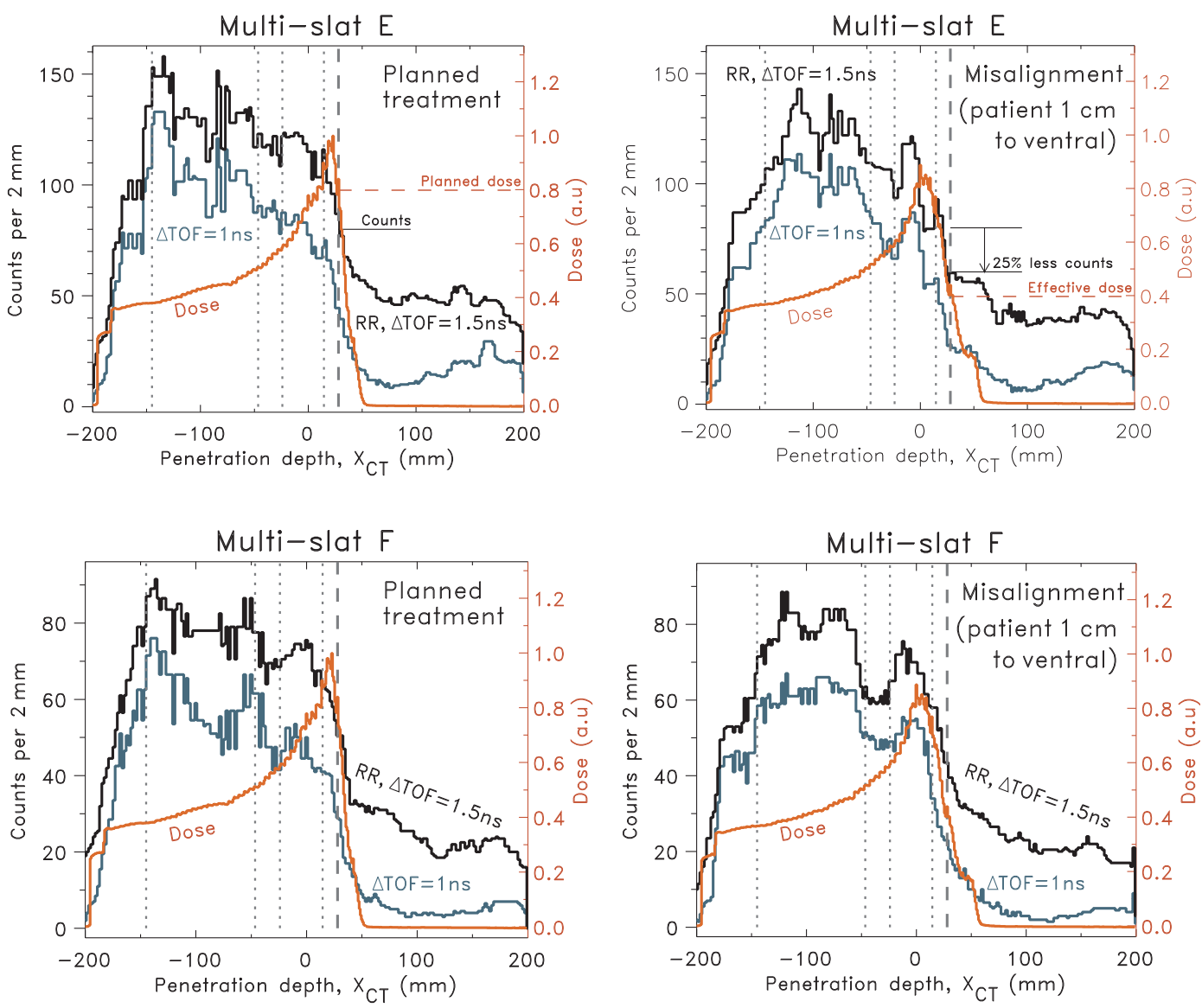

Fig. 14. Collimated prompt gamma profiles simulated for the irradiation of the NCAT prostate (Fig. 12), using multi-slat collimators E and F, and a low-energy threshold of $1 \mathrm{MeV}$. Profiles are shown with ("RR, $\Delta \mathrm{TOF}=1.5 \mathrm{~ns}$ ") and without (" $\Delta \mathrm{TOF}=1 \mathrm{~ns}$ ") considering the beam microstructure. Also shown are the laterallyintegrated depth-dose profiles. A median filter with a span of 8 or 12 bins was applied, for multi-slat collimators $\mathrm{E}$ and $\mathrm{F}$, respectively (bin size $=2 \mathrm{~mm}$ ). The vertical dashed lines correspond to the proton range in the 'Planned treatment' situation. The vertical dotted lines represent the soft-tissue/bone, bone/soft-tissue, soft-tissue/ prostate, and prostate/soft-tissue interfaces traversed by the protons, from left to right, respectively. 
alone, resulting in an enhancement of the distal-falloff steepness, and better visibility of other profile features. Fig. 14-left shows that even with a repetition rate of $100 \mathrm{MHz}$, the distal falloff of the counts profile is steep enough, providing valuable information with agreement to the falloff of the BP. This is not the case in the equivalent profile in Fig. 14right, meaning that a compromised treatment is taking place.

Furthermore, PG profiles show a correlation with certain anatomical features, such as the bone tissue, comprehended between the 1st and 2nd dotted lines.

The profiles obtained with multi-slat E present the best overall visual correlation to the depth-dose and anatomy, in this study. In particular, we can detect about $25 \%$ reduction in the number of counts of the PG profile from the 'misalignment' case (Fig. 14-top), at the expected proton range position, in regard to the 'planned treatment' case, corresponding to a $50 \%$ decrease of the depth-dose value. In practice, the measured PG profiles may be compared with reference simulated ones (based on the treatment planning CT). In addition, measured PG profiles from a given fraction could be compared to those measured in the preceding treatment fractions, when available, to verify consistency or detect important deviations. The same applies for range verification.

\section{Conclusions and outlook}

In this work, we simulated prompt-gamma imaging with various multi-slat collimator configurations placed at different distances from the beam axis, for realistic proton-therapy dose distributions in a human-like, voxelized phantom. The goal of the work was to obtain a multi-slat collimation system capable of providing high detectability to pertinent patient morphological modifications. Such modifications were obtained either from data published in the literature, or from the experience of some of the authors working in particle therapy environments. We compared collimated prompt gamma profiles obtained from the aforementioned compromised treatment deliveries with those from reference (as-planned) irradiations and were able to visually detect relevant indicators of deviations in the delivered dose, in particular the depth-dose distal falloff position and the position of anatomic structures such as bone and air-cavities.

Different multi-slat configurations placed at various distances from the beam axis were compared in terms of photon signal-to-background ratio $(S \gamma / B \gamma)$ and range- and heterogeneity-resolving power. Here, it is to be noted that the performance of a collimator does not only depend on its (intrinsic) geometric performance but also on the $S \gamma / B \gamma$. In all cases, a large amount of background counts (compared to the number of signal counts) was detected. The collimator is exposed to the highest fluence of neutrons (producing background gamma rays within the collimator material) if placed close to the patient. Additional 'uncorrelated' counts, which cannot be rejected by TOF, result from prompt gamma rays that undergo Compton scattering and/or trigger electromagnetic cascades in the collimator material. These results indicate that, due to the high total background level, a collimator with a relatively high geometric efficiency is preferable to a collimator with a relatively high intrinsic resolution, if the collimator is placed closer to the beam. If the same collimator is displaced further away from the beam, its intrinsic resolution deteriorates linearly with the distance (Eq. (1)), whereas its efficiency remains essentially constant (Eq. (2)). However, at larger distances from the beam the background level is lower, allowing for a different trade-off, namely a better resolution. A good spatial resolution is in principle desirable, especially in very heterogeneous targets, to detect changes in the longitudinal PG profile that contain important clues on the (causes of) deviations between the planned and delivered dose distribution.

As seen elsewhere in the literature, it further appears necessary to develop and optimize methods for the suppression of background counts. Background rejection by means of shifting time-of-flight discrimination in combination with a $1 \mathrm{MeV}$ low-energy threshold appeared much more effective than using energy selection only ( $3 \mathrm{MeV}$ threshold). In fact, TOF discrimination appeared crucial to obtain a good $\mathrm{S} \gamma / \mathrm{B} \gamma$ ratio in the pelvic irradiation with $200 \mathrm{MeV}$ protons, due to the increased amount of neutron-induced background from the higher energetic protons and the increased collimator size. Since the width of the TOF window is mainly determined by the proton bunch width, which can be of the order of 1 ns or lower for cyclotron beams at energies comprehended between $160 \mathrm{MeV}$ and $230 \mathrm{MeV}$ (bunch widths between $\sim 1$ ns and 380 ps FWHM, respectively [55]), the analysis made in this paper is realistic. Furthermore, all results concerning PG profiles took into account a cyclotron repetition rate of $100 \mathrm{MHz}$, which represents a standard to worst case scenario. We have shown that even in such situation, a multi-slat detection system is capable of detecting all patient modifications studied in this work (perfect detector).

Gueth et al. [26] have suggested a different multi-slat configuration, made of $10 \mathrm{~cm}$ height and $2 \mathrm{~mm}$ thick tungsten slats with a $2 \mathrm{~mm}$ slit aperture size and with a beam-to collimator distance $b=25 \mathrm{~cm}$. In their concept, the detector is placed $25 \mathrm{~cm}$ behind the collimator back-surface in order to reduce septa shadowing. This requires that the distance between the detector front surface and the beam axis is kept constant, hence their detector has an annular shape in the azimuthal plane. In our view, the space between the back surface of the collimator and the detector leads to undesirable degradation of spatial resolution. Therefore, in the present work we assume a simple flat-panel detector placed immediately behind the collimator, while the problem of septa shadowing is solved by applying a median filter to the recorded PG profile. Indeed, in the optimized multi-slit designs obtained by Pinto et al. [56] the space between the collimator and the detector is kept as low as a few millimeters. Additional advantages of this approach are simplicity of implementation and the fact that the collimator can be used at more than one distance from the beam axis.

Interestingly, our multi-slat configuration $\mathrm{E}$ is rather similar to an optimal multi-slat configuration found in an independent study by Pinto et al. [56], using a complex mathematical algorithm to predict the best precision on the fall-off position in an homogeneous target. This indicated that the simple and comprehensive analysis of geometrical collimator resolution and efficiency used in this work, already provides first-order approximations of appropriate collimator geometries.

In conclusion, the results of this work provide several insights on the optimization of multi-slat collimator design for prompt-gamma imaging in pertinent clinical scenarios. Our results indicate that multi-slat PG imaging could be performed with all clinically used proton energies using a collimator placed at either a small or large distance from the target. This leaves flexibility to adapt the setup to practical and clinical considerations. The work highlights the relative simplicity and versatility of multi-slat PG imaging for in situ particle therapy treatment verification. In addition to enabling in vivo measurement of the proton range, multi-slat PG imaging potentially allows retrieving clues on the anatomical changes and patient setup errors that may occur relative to the treatment plan, thus enabling the detection of large as well as subtle deviations (range deviations as small as $2 \mathrm{~mm}$ ) in the treatment delivery. We note that the value $2 \mathrm{~mm}$ represents the contribution of the collimator and patient scattering to the complete prompt gamma system. Future work shall focus on the implementation and optimization of the scintillation detection system, since several solutions can be implemented. For example, the scintillators can be positioned inside the multi-slat collimator, at the expense of decreased detection efficiency, or a large block of scintillator readout in Anger logic can be used, which increases detection efficiency but decreases spatial resolution. The work of Liprandi et al. [57] has established a spatial resolution inferior to $3 \mathrm{~mm}$ with a monolithic scintillator. However, a study with a segmented scintillator could reveal different (smaller) resolutions. In addition, pulse height versus integral charge readout strategies should also be examined. 


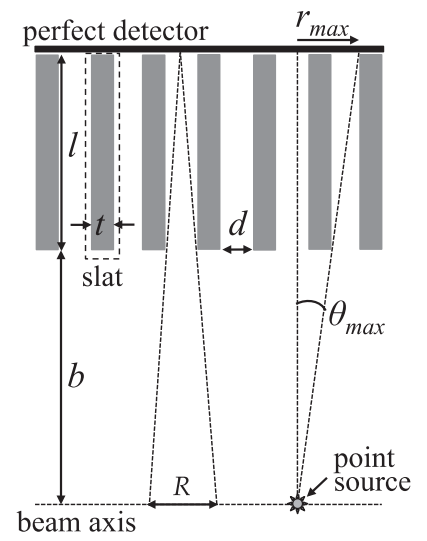

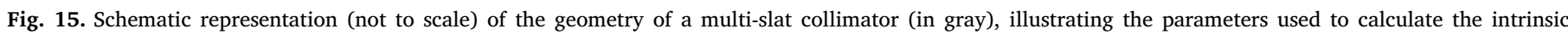
resolution and efficiency of the collimators. $R$ is the intrinsic point position resolution.

\section{Acknowledgements}

This work was funded in part by the Foundation for Fundamental Research on Matter (FOM), The Netherlands (Grant No. 09NIG18). P. Cambraia Lopes was supported by Grant No. SFRH/BD/73705/2010 and P. Crespo was supported by Grant No. SFRH/BPD/39223/2007, both from FCT - Fundação para a Ciência e a Tecnologia, Lisbon, Portugal, co-funded by the European Social Fund and by POPH Programa Operacional Potencial Humano. P. Crespo and H. Simões were also supported by QREN - FEDER under the Sistema de Incentivos à Investigação e Desenvolvimento Tecnológico (CENTRO-07-ST24FEDER-002007, project 'Radiation for Life').
The authors like to thank Paul Segars (Johns Hopkins University) for providing the software-based anthropomorphic NCAT phantom, Marco Pinto (LIP-Coimbra) for the support given on the simulation code, Freek Beekman and Victor Bom (TU Delft) for helpful discussions on collimator design, Julien Smeets (Ion Beam Applications SA, Louvain-laNeuve) for sharing the data simulated with MCNPX on neutron and gamma yields escaping PMMA, and Georgios Dedes (Ludwig Maximilian Universität, Muenchen) for helpful discussions on Geant4 models. Finally, thanks for the informatics support given by the staff of the Milipeia (University of Coimbra) and HPC11 (TU Delft) high-performance computer clusters.

\section{Appendix A}

In this appendix we derive Eq. (2) that gives the geometrical efficiency of a multi-slat collimator. Given the beam-azimuthal symmetry, we restrict this analysis to two dimensions, i.e. in the plane defined by the beam axis and the normal to the detector plane (Fig. 15). This is a special case of the classical 3-dimensional analysis of parallel-hole collimators described in Cherry et al. (2003, chapter 14).

The fraction of gamma rays transmitted through the collimator from a point source on the beam axis is derived. We assume a perfect (i.e. infinitely dense) collimator. The point source position on the beam axis for a multi-slat collimator is arbitrary, as the efficiency is constant throughout the collimator field-of-view. Let $\theta$ be the angle between the 2-dimensional photon momentum and the slat planes (Fig. 15). If $\theta=0$, i.e. if the photon momentum is normal to the beam direction, the probability $\alpha$ for the photon to be transmitted depends only on the slit aperture size $d$ and the slat/septa thickness $t$, according to Eq. (A.1):

$\alpha=d /(d+t)$

For obliquely incident photons (i.e. $\theta \neq 0$ ) the probability of transmission decreases as $|\theta|$ increases, and it equals zero for $|\theta| \geq \theta_{\max }$, with $\theta_{\max }$ defined by $\tan \left(\theta_{\max }\right)=d / l$, in which $l$ is the collimator height.

Let $r$ be defined as the distance on the detector surface (in the beam-axis direction, Fig. 15) between the point of incidence of an oblique photon and a normally incident photon emitted by the same point source, such that:

$\left\{\begin{array}{c}r=(l+b) \cdot \tan (\theta) \\ r_{\max }=(l+b) \cdot(d / l)\end{array}\right.$

where $b$ is the source-to-collimator distance. The probability of transmission of a gamma ray incident at $r$ can be approximated by a linear function, $f(r)$, where $f(0)=\alpha$ and $f\left(r_{\max }\right)=0$ :

$f_{m-s}(r)=\left\{\begin{array}{c}\alpha\left[1-\frac{r}{r_{\max }}\right], 0 \leq r \leq r_{\max } \\ 0, r>r_{\max }\end{array}\right.$

Assuming symmetry around $r=0$, i.e. $f(-r)=f(r)$, the 2D geometric efficiency of the multi-slat collimator can now be written as:

$g^{\text {multi-slat }}=2 \cdot \int_{0}^{r_{\max }} \frac{f_{m-s}(r)}{2 \pi(l+b)} \mathrm{d} r$

where $(l+b)$ is the source-to-detector distance and $\mathrm{d} r /[2 \pi(l+b)]$ is the elemental angular coverage for a direction of incidence with transmission probability $f_{m-s}(r)$. By substituting Eqs. (A.1) and (A.3) in Eq. (A.4) and solving the integral function, we obtain:

$g^{\text {multi-slat }}=\frac{d}{(d+t) \pi(l+b)} \int_{0}^{r_{\max }} 1-\frac{r}{r_{\max }} \mathrm{d} r=\frac{d}{(d+t) \pi(l+b)} \cdot\left[r-\frac{r^{2}}{2 r_{\max }}\right]_{0}^{r_{\max }}=\frac{d}{(d+t) \pi(l+b)} \cdot \frac{r_{\max }}{2}$ 
Finally, by substituting Eq. (A.2) for $r_{\max }$, the 2D geometric efficiency of the multi-slat collimator is given by:

$g^{\text {multi-slat }}=\frac{d}{(d+t) \pi(l+b)} \cdot \frac{(l+b)}{2} \cdot \frac{d}{l}=\frac{d^{2}}{2 \pi l(d+t)}$, with $l>d^{2} /[2 \pi(d+t)]$

\section{References}

[1] Wilson R. Radiological use of fast protons. Radiology 1946;47(11):487-91.

[2] Engelsman M, Schwarz M, Dong L. Physics controversies in proton therapy. Semin Radiat Oncol 2013;23(2):88-96.

[3] Schaffner B, Pedroni E. The precision of proton range calculations in proton radiotherapy treatment planning: experimental verification of the relation between CT-HU and proton stopping power. Phys Med Biol 1998;43:1579-92.

[4] Cameron SJ, Grant IWB, Lutz W, Pearson JG. The early effect of irradiation on ventilatory function in bronchial carcinoma. Clin Radiol 1969;20(1):12-8.

[5] Nishio T, Ogino T, Nomura K, Uchida H. Dose-volume delivery guided proton therapy using beam on-line PET system. Med Phys 2006;33(11):4190-7.

[6] Stichelbaut F, Jongen Y. Verification of the proton beam position in the patient by the detection of prompt gamma-rays emission. In: 39th Meeting of the Particle Therapy Co-Operative Group (San Francisco, CA); 2003.

[7] Min CH, Kim CH, Youn MY, Kim JW. Prompt gamma measurements for locating the dose falloff region in the proton therapy. Appl Phys Lett 2006;89:183517.

[8] Enghardt W, Crespo P, Fiedler F, Hinz R, Parodi K, Pawelke J, et al. Charged hadron tumour therapy monitoring by means of PET. Nucl Instrum Methods Phys Res, Sect A 2004;525:284-8.

[9] Crespo P, Shakirin G, Enghardt W. On the detector arrangement for in-beam PET for hadron therapy monitoring. Phys Med Biol 2006;51:2143-63.

[10] Parodi K, Paganetti H, Shih HA, Michaud S, Loeffler JS, DeLaney TF, et al. Patient study of in vivo verification of beam delivery and range, using positron emission tomography and computed tomography imaging after proton therapy. Int J Radiat Oncol Biol Phys 2007;68(3):920-34.

[11] Nishio T, Miyatake A, Ogino T, Nakagawa K, Saijo N, Esumi H. The development and clinical use of a beam on-line system mounted on a rotating gantry port in proton therapy. Int J Radiat Oncol Biol Phys 2010;76(1):277-86.

[12] Kang BH, Kim JW. Monte Carlo design study of a gamma detector system to locate distal dose falloff in proton therapy. IEEE Trans Nucl Sci 2009;56:46-50.

[13] Richard $\mathrm{MH}$, et al. Design guidelines for a double scattering Compton camera for prompt gamma imaging during ion beam therapy: a Monte Carlo simulation study. IEEE Trans Nucl Sci 2011;58:87-94.

[14] Peterson SW, Robertson D, Polf J. Optimizing a three-stage Compton camera for measuring prompt gamma rays emitted during proton radiotherapy. Phys Med Biol 2010;55:6841-56.

[15] Kormoll T, et al. Compton imager for in-vivo dosimetry of proton beams: a design study. Nucl Instrum Methods Phys Res, Sect A 2011;626(7):114-9.

[16] Verburg JM, Seco J. Proton range verification through prompt gamma-ray spectroscopy. Phys Med Biol 2014;59:7089-106.

[17] Golnik C, et al. Range assessment in particle therapy based on prompt $\gamma$-ray timing measurements. Phys Med Biol 2014;59:5399-422.

[18] Polf JC, Peterson S, McCleskey M, Roeder BT, Spiridon A, Beddar S, et al. Measurement and calculation of characteristic prompt gamma ray spectra emitted during proton irradiation. Phys Med Biol 2009;54:N519-27.

[19] Polf JC, Panthi R, Mackin DS, McCleskey M, Saastamoinen A, Roeder BT, et al. Measurement of characteristic prompt gamma rays emitted from oxygen and carbon in tissue-equivalent samples during proton beam irradiation. Phys Med Biol 2013;58:5821-31.

[20] Verburg JM, Riley K, Bortfeld T, Seco J. Energy- and time-resolved detection of prompt gamma-rays for proton range verification. Phys Med Biol 2013;58:L37-49.

[21] Kelleter L, Wrońska A, Besuglow J, Konefał A, Laihem K, Leidner J, et al. Spectroscopic study of prompt-gamma emission for range verification in proton therapy. Phys Med 2017;34:7-17.

[22] Zarifi M, Guatelli S, Bolst D, Hutton B, Rosenfeld A, Qi Y. Characterization of prompt gamma-ray emission with respect to the Bragg peak for proton beam range verification: a Monte Carlo study. Phys Med 2017;33:197-206.

[23] Biegun AK, Seravalli E, Cambraia Lopes P, Rinaldi I, Pinto M, Oxley DC, et al. Timeof-flight neutron rejection to improve prompt-gamma-ray imaging for real-time proton range verification: a simulation study. Phys Med Biol 2012;57:6429-44.

[24] Min CH, Lee HR, Yeom YS, Cho S, Kim CH. Determination of the distal dose edge in a human phantom by measuring the prompt gamma distribution: a Monte Carlo study. J Korean Phys Soc 2010;56(6):2059-62.

[25] Min CH, Lee HR, Kim CH, Lee SB. Development of array-type prompt gamma measurement system for in vivo range verification in proton therapy. Med Phys 2012;39(5):2100-7.

[26] Gueth P, et al. Machine learning-based patient specific prompt-gamma dose monitoring in proton therapy. Phys Med Biol 2013;58:4563-77.

[27] Kim D, Yim H, Kim J-W. Pinhole camera measurements of prompt gamma-rays for detection of beam range variation in proton therapy. J Korean Phys Soc 2009;55(4):1673-6.

[28] Peloso R, Busca P, Fiorini C, Basilavecchia M, Frizzi T, Smeets J, et al. Application of the HICAM camera for imaging of prompt gamma rays in measurements of proton beam range. In: Nucl. Sci. Symp. Conf. Record (NSS/MIC) 2011; p. 2285-89.

[29] Bom V, Joulaeizadeh L, Beekman F. Real-time prompt gamma monitoring in spotscanning proton therapy using imaging through a knife-edge-shaped slit. Phys Med Biol 2012:57:297-308.

[30] Smeets J, Roellinghoff F, Prieels D, Stichelbaut F, Benilov A, Busca P, et al. Prompt gamma imaging with a slit camera for real time range control in proton therapy. Phys Med Biol 2012;57:3371-405.

[31] Testa E, Bajard M, Chevallier M, Dauvergne D, Le Foulher F, Freud N, et al. Dose profile monitoring with carbon ions by means of prompt-gamma measurements. Nucl Instrum Methods Phys Res, Sect B 2009. 267.993-6.

[32] Testa M, Bajard M, Chevallier M, Dauvergne D, Freud N, Henriquet P, et al. Realtime monitoring of the Bragg-peak position in ion therapy by means of single photon detection. Radiat Environ Biophys 2010;49:337-43.

[33] Roellinghoff F, Benilov A, Dauvergne D, Dedes G, Freud N, Janssens G, et al. Realtime proton beam range monitoring by means of prompt-gamma detection with a collimated camera. Phys Med Biol 2014;59:1327-38.

[34] Smeets J, Roellinghoff F, Janssens G, Perali I, Celani A, Fiorini C, et al. Experimental comparison of knife-edge and multi-parallel slit collimators for prompt gamma imaging of proton pencil beams. Front Oncol 2016;6(156):1-8.

[35] Xie Y, Bentefour H, Janssens G, Smeets J, Stappen FV, Hotoiu L, et al. A and Teo BKK. Prompt gamma imaging for in vivo range verification of pencil beam scanning proton therapy. Int J Radiat Oncol Biol Phys 2017;99(1):210-8.

[36] Richter C, Pausch G, Barczyk S, Priegnitz M, Keitz I, Thiele J, et al. First clinical application of a prompt gamma based in vivo proton range verification system. Radiother Oncol 2016;118(2):232-7.

[37] Krimmer J, Dauvergne D, Létang JM, Testa E. Prompt-gamma monitoring in hadrontherapy: a review. Nucl Instrum Methods Phys Res, Sect A 2018;878:58-73.

[38] Segars WP. Development of a new dynamic NURBS-based cardiac-torso (NCAT) phantom PhD. dissertation The University of North Carolina; 2001.

[39] Cambraia Lopes P, Pinto M, Simões H, Biegun AK, Dendooven P, Oxley DC, et al. Optimization of collimator designs for real-time proton range verification by measuring prompt gamma rays. In: 2012 IEEE Nucl. Sci. Symp. Med. Imaging Conf Record (NSS/MIC) 2012; p. 3864-70.

[40] Cherry SR, Sorenson JA, Phelps ME. Physics in Nuclear Medicine. Elsevier, PA: Saunders; 2003 chapter 14.

[41] Hubbell JH, Seltzer SM. X-Ray Attenuation and Absorption for materials of Dosimetric Interest NIST Standard Reference Database 126 National Institute of Standards and Technology (NIST) Physical Meas. Laboratory; 2004.

[42] Agostinelli S, et al. Geant4: a simulation toolkit. Nucl Instrum Methods Phys Res, Sect A 2003;506(3):250-303.

[43] Allison J, et al. Geant4 developments and applications. IEEE Trans Nucl Sci 2006;53(1):270-8.

[44] Crespo P, Reis J, Couceiro M, Blanco A, Ferreira NC, Ferreira Marques R, et al. Whole-body single-bed time-of-flight RPC-PET: simulation of axial and planar sensitivities with NEMA and anthropomorphic phantoms. IEEE Trans Nucl Sci 2012;59(3):520-9.

[45] Schwaab J, Brons S, Fieres J, Parodi K. Experimental characterization of lateral profiles of scanned proton and carbon ion pencil beams for improved beam models in ion therapy treatment planning. Phys Med Biol 2011;56:7813-27.

[46] Parodi K, Mairani A, Brons S, Hasch BG, Sommerer F, Naumann J, et al. Monte Carlo simulations to support start-up and treatment planning of scanned proton and carbon ion therapy at a synchrotron-based facility. Phys Med Biol 2012;57:3759-84.

[47] Verburg JM, Shih HA, Seco J. Simulation of prompt gamma-ray emission during proton radiotherapy. Phys Med Biol 2012;57:5459-72.

[48] Robert C, et al. Distributions of secondary particles in proton and carbon-ion therapy: a comparison between GATE/Geant4 and FLUKA Monte Carlo codes. Phys Med Biol 2013;58:2879-99.

[49] Dedes G, Pinto M, Dauvergne D, Freud N, Krimmer J, Létang JM, et al. Assessment and improvements of Geant4 hadronic models in the context of prompt-gamma hadrontherapy monitoring. Phys Med Biol 2014;59:1747-72.

[50] Janssen FMFC, Landry G, Cambraia Lopes P, Dedes G, Smeets J, Schaart DR, et al Factors influencing the accuracy of beam range estimation in proton therapy using prompt gamma emission. Phys Med Biol 2014;59:4427-41.

[51] Denham JW, Hauer-Jensen M. The radiotherapeutic injury - a complex 'wound' Radiother Oncol 2002;63:129-45.

[52] Cambraia Lopes P, Clementel E, Crespo P, Henrotin S, Huizenga J, Janssens G, et al. Time-resolved imaging of prompt-gamma rays for proton range verification using a knife-edge slit camera based on digital photon counters. Phys Med Biol 2015;60:6063-85.

[53] Badziak J. Laser-driven ion acceleration: methods, challenges and prospects. J Phys Conf Ser 2018;959(1):012001.

[54] Frey K, Unholtz D, Bauer J, Debus J, Min CH, Bortfeld T, et al. Automation and uncertainty analysis of a method for in-vivo range verification in particle therapy. Phys Med Biol 2014;59:5903.

[55] Hueso-Gonzalez F, Enghardt W, Fiedler F, Golnik C, Janssens G, Petzoldt J, et al. First test of the prompt gamma ray timing method with heterogeneous targets at a clinical proton therapy facility. Phys Med Biol 2015;60:6247.

[56] Pinto M, Dauvergne D, Freud N, Krimmer J, Letang JM, Ray C, et al. Design optimisation of a TOF-based collimated camera prototype for online hadrontherapy monitoring. Phys Med Biol 2014;59:7653-74.

[57] Liprandi S, Mayerhofer M, Aldawood S, Binder T, Dedes G, Miani A, et al. Sub-3mm spatial resolution from a large monolithic $\mathrm{LaBr} 3(\mathrm{Ce})$ scintillator. Curr Directions Biomed Eng 2017;3(2):655-9. 